JOURNAL OF THE

AMERICAN MATHEMATICAL SOCIETY

Volume 18, Number 3, Pages 571-603

S 0894-0347(05)00489-3

Article electronically published on April 27, 2005

\title{
IMBEDDED SINGULAR CONTINUOUS SPECTRUM FOR SCHRÖDINGER OPERATORS
}

\author{
ALEXANDER KISELEV
}

\section{INTRODUCTION}

Let $H_{V}$ denote the one-dimensional Schrödinger operator defined on the half-axis by the differential expression

$$
H_{V}=-\frac{d^{2}}{d x^{2}}+V(x)
$$

and some self-adjoint boundary condition at zero. The operator (1.1) describes a charged particle, such as an electron, in the electric field $V(x)$. When $V(x)$ is decaying quickly, one expects the spectral and dynamical properties of $H_{V}$ to remain close to those of the free operator $H_{0}$. Recall that with the operator (1.1) one can associate in a canonical way a spectral measure $\mu$ (see, for example, [6, 2]) which contains much information about the quantum system. The classical result in this direction, going back to the beginning of the century, is that if $V \in L^{1}$, the spectral measure on the positive semi-axis remains purely absolutely continuous. In one dimension, an absolutely continuous spectrum corresponds to the ballistic rate of propagation of the particle. A natural question to ask is what are the critical rates of decay at which some changes in the spectral and dynamical properties of $H_{V}$ may happen. As far as $V(x)$ has any decay at all, the essential spectrum coincides with $[0, \infty)$, but the quality of the spectrum and dynamics may change. In 1928, Wigner and von Neumann [27] showed that there exist potentials $V(x)$ satisfying $|V(x)| \leq \frac{C}{x+1}$ such that $H_{V}$ has positive imbedded eigenvalue $E=1$. This is a purely quantum resonance phenomenon, since $V$ can be chosen arbitrarily small and the bound state originates from long-range correlations in the potential rather than the usual confining effect. Naboko [17] and Simon [25] provided constructions which show that much more drastic changes are possible if the potential decays arbitrarily slower than Coulomb. Namely, for any positive function $h(x)$ which grows at infinity, there exist potentials $V$ such that $|V(x)| \leq \frac{h(x)}{1+x}$ and $H_{V}$ has a dense set of positive eigenvalues. Due to some recent results [3, 21], it is known that the absolutely continuous spectrum on the positive semi-axis is preserved for $|V(x)| \leq C(1+x)^{-1 / 2-\epsilon}$ and in fact for $V \in L^{2}$ [8]. More precisely, for such potentials the absolutely continuous part of the spectral measure, $\mu^{\text {ac }}$, gives positive weight to any subset of $(0, \infty)$ of positive Lebesgue measure. Therefore, the Naboko

Received by the editors November 14, 2003.

2000 Mathematics Subject Classification. Primary 34L40; Secondary 34L25.

Key words and phrases. Schrödinger operators, scattering, singular spectrum.

The author was supported in part by NSF grant DMS-0314129 and by an Alfred P. Sloan Research Fellowship. 
and Simon examples have a dense point spectrum imbedded into the absolutely continuous spectrum.

In [24], Simon has posed fifteen problems on Schrödinger operators, one of which asks whether Schrödinger operators with potentials satisfying $|V(x)| \leq C(1+$ $|x|)^{-1 / 2-\epsilon}$ can also have an imbedded singular continuous spectrum. Remling [21] proved that if $V(x)=o(1 / x)$, then the singular continuous spectrum is empty. Remling [22] and Christ-Kiselev [4] provided estimates on the dimension of the support of the possible singular component of the measure for $|V(x)| \leq C(1+x)^{-\alpha}$. Remling 23] and Kriecherbauer-Remling [13] constructed power-decaying potentials which have decaying solutions on the sets of Hausdorff dimension exactly as predicted by 22, 4. However these constructions are not sufficient to infer the presence of the singular continuous component. The problem of controlling a singular continuous spectrum is difficult since one has to study a set of energies which is uncountable but has Lebesgue measure zero. Because of that, in most known situations, the existence of a singular continuous spectrum is proven "not by what it is, but by what it is not": by elimination of possibilities for having an absolutely continuous or point spectrum. For instance, see the classical example of Pearson [18 or the genericity of the singular continuous spectrum results of Simon [26. However, when the singular continuous spectrum is imbedded, such a path is obviously ruled out. We notice that the fact that potentials leading to operators with an imbedded singular continuous spectrum exist follows from the inverse spectral theory (see, e.g. [15, 16]). Recently, Denisov [9] gave the first proof of existence of decaying potentials leading to an imbedded singular continuous spectrum. Killip and Simon [1] subsequently proved a beautiful theorem establishing necessary and sufficient conditions for a measure to be a spectral measure of a free Jacobi matrix with $l^{2}$ perturbation. In this paper, we are going to give a fairly explicit construction of potentials leading to an imbedded singular continuous spectrum which works under sharp assumptions on the rate of decay. In particular, examples with any power decay slower than $x^{-1}$ follow. The situation in this problem is somewhat parallel to Fourier transforms of measures, where it is easier to relate continuity properties of the measure to the average decay of the Fourier transform, but results and examples regarding pointwise decay are harder to obtain. Moreover, to the best of our knowledge, up until now there were no constructive examples of Schrödinger operators with an imbedded singular continuous spectrum in any setting.

There is an additional point of view on the problem of an imbedded singular continuous spectrum for potentials which decay faster than $L^{2}$, which in our opinion makes the question even more interesting. Recall the definition of wave operators

$$
\Omega_{ \pm} f=s-\lim _{t \rightarrow \mp \infty} e^{i H_{V} t} e^{-i H_{0} t} f
$$

where the limit is taken in the strong $L^{2}$ sense. Existence of wave operators implies that the absolutely continuous spectrum of $H_{V}$ fills the whole positive semi-axis and provides much more precise information about long-time perturbed dynamics. The wave operators are called asymptotically complete (see, e.g. [20]) if the range of $\Omega_{ \pm}$coincides with the orthogonal complement of the subspace spanned by eigenvectors of the operator $H_{V}$. An alternative equivalent characterization is that the range of the wave operators is equal to the absolutely continuous subspace $\mathcal{H}_{\mathrm{ac}}\left(H_{V}\right)$ of the operator $H_{V}$, and that the singular continuous spectrum $\sigma_{\mathrm{sc}}\left(H_{V}\right)$ is empty. 
The intuitive meaning of asymptotic completeness is that the dynamics of the perturbed operator can be divided into two well-understood parts: scattering states travelling to infinity in a way similar to the free evolution, and bound states which remain confined in a certain sense for all times. On the other hand, the singular continuous spectrum is less well understood and corresponds to an intermediate situation: states which do not remain bounded in time, but travel slower, may exhibit recurrent phenomena and do not look like scattering states (see, for example, [14] and the discussion in [5] for a more detailed explanation and further references). A significant effort in quantum mechanical scattering theory was devoted to proving asymptotic completeness, and thus the absence of a singular continuous spectrum, in a wide variety of situations (see, e.g. 7] for a review of some examples). However, to the best of our knowledge, there were no examples of Schrödinger operators for which wave operators exist but are not asymptotically complete due to the presence of a singular continuous spectrum. In the problem that we consider, a classical result is the existence of wave operators for $V \in L_{1}$, and in this class wave operators are also asymptotically complete. However, recently in [5], the existence of (modified) wave operators for $V \in L_{p}, p<2$ was established. Moreover, the usual Möller wave operators (1.2) exist if the $\operatorname{limit}_{\lim } \rightarrow \infty \int_{0}^{x} V(y) d y$ exists. Therefore, an example of a (conditionally integrable) potential in $L^{p}, p<2$ with imbedded singular continuous spectrum would also provide the first known example of a Schrödinger operator for which wave operators exist but fail to be asymptotically complete due to the presence of the singular continuous spectrum.

Our main goal in this paper is to prove the following.

Theorem 1.1. Given any positive function $h(x)$ tending to infinity as $x$ grows, there exist potentials $V(x)$ such that $|V(x)| \leq \frac{h(x)}{1+x}$ and the operator $H_{V}$ has a non-empty singular continuous spectrum.

It is easy to see that without loss of generality one can assume that $h(x)$ is monotone. For the rest of the paper, we are going to operate under this assumption, which is convenient for technical reasons. We are going to provide a fairly explicit construction of such potentials. As a corollary of such a construction we are also going to prove

Theorem 1.2. Given any positive function $h(x)$ tending to infinity as $x$ grows, there exist potentials $V(x)$ such that $|V(x)| \leq \frac{h(x)}{1+x}$, and the wave operators for the operator $H_{V}$ exist but are not asymptotically complete.

Our examples can be easily generalized to any dimension by looking at spherically symmetric potentials.

Finally, we will prove

Theorem 1.3. Suppose that the potential $V(x)$ satisfies $|V(x)| \leq B(1+x)^{-1}$. Then the singular continuous spectrum of the operator $H_{V}$ is empty.

This result shows that our construction is sharp. For potentials decaying at the Coulomb rate, the (modified) wave operators are necessarily asymptotically complete. The best previously known result in this direction is that the singular continuous spectrum is absent if $V(x)=o(1 / x)$ [21. 
Let us outline briefly the idea of the construction leading to Theorem 1.1. Controlling a singular continuous spectrum is hard because unlike a point spectrum, one has to control in a certain sense an uncountable set of energies, and there is no simple criteria (like $L^{2}$ ) for what behavior of solutions implies the existence of a singular continuous component. It turns out that one can reduce matters to keeping track at each step of only a finite number of energies, but the price one has to pay is having to control rather precisely the norms of the solutions at these energies. The control has to be much more precise than in the constructions of Naboko and Simon. At the heart of the construction is a lemma which allows us instead of one imbedded eigenvalue in the Wigner-von Neumann-type construction to get two, each having an eigenfunction (normalized at the origin) with the $L^{2}$ norm squared being exactly twice the $L^{2}$ norm squared of the eigenfunction in a usual Wigner-von Neumann construction in a certain asymptotic regime. The $L^{2}$ norm squared of the eigenfunction is the inverse of the point weight the measure $\mu$ assigns to a given eigenvalue. Thus the lemma will allow us to "spread out" evenly the weight of one point mass to two nearby points. This allows us to carry out a Cantor-like construction, building a sequence of potentials which have point measure components approximating a singular continuous measure. In the limit, one gets the result.

\section{A SPlitTing Lemma}

For the rest of the paper, let us fix for simplicity some boundary conditions at the origin, for example Dirichlet. Let us denote by $\mu$ the spectral measure corresponding to this boundary condition. Throughout the paper, we are going to use the notation $C$ for universal constants (not necessarily the same), more precisely, for the constants which do not depend in any way on the step of the inductive construction. A starting point is the following elementary observation.

Lemma 2.1. Let $E$ be an eigenvalue of $H_{V}$, and $u(x, E)$ be the corresponding eigenfunction normalized by the condition $u^{\prime}(0, E)=1$. Then $\mu(E)=\|u(x, E)\|_{L^{2}\left(\mathbb{R}^{+}\right)}^{-2}$.

Proof. Denote by $v(x, E)$ the solution satisfying the orthogonal boundary condition $v(x, E)=1, v^{\prime}(x, E)=0$. Recall that the Weyl $m$-function is defined for $z \in \mathbb{C} \backslash \mathbb{R}$ as a unique complex number $m(z)$ such that $v(x, z)+m(z) u(x, z) \in L^{2}$ (assuming we are in the limit point case). We have (see e.g. [6], Chapter 9, Section 3)

$$
m(z)-m\left(z_{0}\right)=\int_{\mathbb{R}}\left(\frac{1}{t-z}-\frac{1}{t-z_{0}}\right) d \mu(t),
$$

where $z_{0}$ is some fixed complex number. From (2.1) it follows that

$$
\mu(E)=\lim _{\epsilon \rightarrow 0} \epsilon|m(E+i \epsilon)|=\lim _{\epsilon \rightarrow 0} \epsilon \operatorname{Im} m(E+i \epsilon) .
$$

On the other hand,

$$
\operatorname{Im} m(E+i \epsilon)=\epsilon\|v(x, E+i \epsilon)+m(E+i \epsilon) u(x, E+i \epsilon)\|_{L^{2}\left(\mathbb{R}^{+}\right)}^{2}
$$


(see, e.g. [6]). Multiplying both sides of the above equality by $\epsilon$ and passing to the limit $\epsilon \rightarrow 0$, we get

$$
\mu(E)=\mu(E)^{2}\|u(x, E)\|_{L^{2}}^{2},
$$

which is exactly the claim of the lemma.

Given the energy $E=k^{2}$, it will be convenient to introduce Prüfer variables $R$ and $\theta, R^{2}=\left(u^{\prime}\right)^{2}+k^{2} u^{2}$ and $\theta=\tan ^{-1}\left(k u / u^{\prime}\right)$. Then it is easy to see that

$$
\begin{aligned}
\left(\log R(x, k)^{2}\right)^{\prime} & =\frac{1}{k} V(x) \sin 2 \theta(x, k), \\
\theta(x, k)^{\prime} & =k-\frac{1}{k} V(x)(\sin \theta)^{2} .
\end{aligned}
$$

There are three essential ideas which play an important role in the result: the Splitting Lemma, a relation between the $L^{2}$ norm of $R$ and the derivative of the Prüfer angle $\theta$ in energy, and separation of scales, which allows one to control errors effectively. The main new ingredient is the Splitting Lemma, which reduces to the study of a certain system of nonlinear differential equations. The purpose of this section is to prove this first key lemma, which is at the heart of the construction. To clarify its meaning, let us formulate first a simplified version of this result. Fix an arbitrary positive energy $k_{0}$ (we are going to work with values of the quasimomentum $k$ throughout the paper, but still call those "energies" for simplicity). Fix a parameter $g$, which we think of as large. Define $\tilde{g}=\exp \left(g^{3 / 4}\right)$. Fix another parameter, $f$, which we think of as small. (It is useful to keep in mind that in the actual construction, we will be able to choose $g$ as large as we want and then after that to choose $f$ as small as we want.) Let $\delta k=\tilde{g}^{-1} f$, and denote by $k_{1}, k_{2}$, $k_{2}>k_{1}$, the ends of the interval of length $\delta k$ centered at $k_{0}$. Define $\theta_{i}(x)=\theta\left(x, k_{i}\right)$ and $\delta \theta=\theta_{2}-\theta_{1}$. Furthermore, define a small angle $\alpha$ by a condition $f \sin \alpha=\delta k$. We are going to use the notation

$$
x_{\beta}=\min \{x: \delta \theta(x)=\beta\} .
$$

Define a potential $V(x)$ on $[0, \infty)$ by

$$
V(x)= \begin{cases}-2 f k_{0} \sin \left(\theta_{1}+\theta_{2}\right), & 0 \leq x \leq x_{\pi-\alpha} \\ 2 f k_{0} \sin \left(\theta_{1}+\theta_{2}\right), & x_{\pi-\alpha} \leq x \leq f^{-1} g, \\ -\min \left(\delta k, \frac{g}{2 x}\right) \frac{k_{0}}{4}\left(\sin 2 \theta_{1}+\sin 2 \theta_{2}\right), & x>f^{-1} g .\end{cases}
$$

The definition of $V$ involves the Prüfer angles $\theta_{i}$, and $\theta_{i}$ in their turn depend on $V$ through (2.3). This ambiguity is easily resolved, however, by substituting the expression (2.4) for $V$ into (2.3) and solving the resulting nonlinear system for $\theta_{i}$. It is not difficult to see from (2.3) that this resulting system has a unique piecewise smooth global solution by a standard ODE existence and uniqueness theorem. The functions $\theta_{i}(x)$ are smooth apart from three points where $V$ is not smooth, and where $\theta_{i}^{\prime}\left(\right.$ or $\theta_{i}^{\prime \prime}$ ) may jump. We can then define $V$ in terms of these $\theta_{i}$ by the (2.4) recipe, and then solutions of (2.3) will coincide with $\theta_{i}$ by uniqueness. For the rest of the paper, we are going to consider at each step $V$ defined in terms of Prüfer angles at a finite number of energies without further explanation. We are going to ignore for now the issue whether $V$ is well-defined in a sense that $x_{\pi-\alpha}<f^{-1} g$; this will be verified later for all $g$ large enough. We notice that the constant $f$ in 
(2.4) can be replaced by an appropriate slowly varying function with no difference for the result. We choose a particular representation (2.4) to simplify technicalities. Then we have

Lemma 2.2. Assume that $g$ is sufficiently large, and $f$ is sufficiently small. Then for a potential $V(x)$ given by (2.4), both $k_{1}$ and $k_{2}$ are eigenvalues. Moreover,

$$
\left\|R\left(x, k_{i}\right)\right\|_{L^{2}\left(\mathbb{R}^{+}\right)}^{2}=2 f^{-1}\left(1+O\left(f e^{g}, \tilde{g}^{-1 / 2} g\right)\right)
$$

provided that $R\left(x, k_{i}\right)$ are normalized by a condition $R\left(0, k_{i}\right)=1, i=1,2$.

We are not going to prove Lemma 2.2, but directly a more advanced version which is going to be needed in the construction. The difference is that we will need to handle not just a pair of eigenvalues, but $2^{n-1}$ pairs of eigenvalues at the $n$th step simultaneously. However, we are going to use this more transparent formulation to make a few clarifying remarks. First of all, observe that $|V(x)| \leq h(x) /(x+1)$ provided that $f$ is sufficiently small. Indeed, we basically need to ensure that

$$
2 k_{0} f \leq h\left(f^{-1} g\right) /\left(f^{-1} g+1\right)
$$

or $2 k_{0}(g+f) \leq h\left(f^{-1} g\right)$. This clearly holds if for fixed $g$ we take $f$ sufficiently small. Next, consider a Wigner-von Neumann-type potential $V_{0}(x)$ defined by

$$
V_{0}(x)= \begin{cases}-2 k_{0} f \sin 2 \theta_{0}, & 0 \leq x \leq f^{-1} g \\ -\min \left(\delta k, \frac{g}{2 x}\right) \frac{k_{0}}{2} \sin 2 \theta_{0}, & x>f^{-1} g .\end{cases}
$$

It is fairly straightforward to check using (2.2), (2.3) that $V_{0}$ leads to an eigenvalue at $k_{0}$ with $\left\|R\left(x, k_{0}\right)\right\|_{L^{2}\left(\mathbb{R}^{+}\right)}^{2}=f^{-1}\left(1+O\left(f g, e^{-g}\right)\right)$. Thus Lemma 2.2 provides a way, using a potential satisfying the same upper bound as $V_{0}$, to split the eigenvalue $k_{0}$ into two nearby eigenvalues with equal weights without loss of the total weight (in the asymptotic regime). The construction is not trivial: for example, taking $V=\frac{1}{2}\left(V_{1}+V_{2}\right)$, where $V_{i}$ is given by the same expression as $V_{0}$ at $k_{i}$, leads to a loss of a constant factor in the total mass of two eigenvalues and thus does not work for the Splitting Lemma. Moreover, one cannot hope for a coefficient smaller than 2 in front of $f^{-1}$ in (2.5). As the construction below will show, that would have led by splitting to spectral measures giving arbitrary large weight to a fixed finite interval. But the spectral measure $\mu$ is well known to satisfy $\int \frac{d \mu(t)}{1+|t|^{2}}<C$, with $C$ uniform for uniformly bounded $V$. Therefore, the factor 2 in the Splitting Lemma is optimal.

After these preliminary remarks, let us consider the general case. At the $n$th step of our construction, we are going to look at $2^{n}$ energies $k_{j}^{(n)}, j=1, \ldots, 2^{n}$, ordered in increasing order. We are going to assume that for every $n$, all these energies lie in a fixed compact interval away from zero; moreover, we are going to assume that

$$
2 \max _{j, j^{\prime}}\left|k_{j}^{(n)}-k_{j^{\prime}}^{(n)}\right| \leq \min \left(k_{j}^{(n)}\right)
$$

for every $j, n$. This assumption is made for technical convenience and will be easy to satisfy at each step of the inductive construction. We define $\theta_{j}^{(n)}=\theta\left(x, k_{j}^{(n)}\right)$, $R_{j}^{(n)}=R\left(x, k_{j}^{(n)}\right), \delta k_{j}^{(n)}=k_{2 j}^{(n)}-k_{2 j-1}^{(n)}$, and $\delta \theta_{j}^{(n)}=\theta_{2 j}^{(n)}-\theta_{2 j-1}^{(n)}$. Then $V^{(n)}(x)$ is 
going to be defined by $V^{(n)}(x)=\sum_{j=1}^{2^{n-1}} V_{j}^{(n)}(x)$, where

$$
V_{j}^{(n)}(x)=\left\{\begin{array}{l}
-2 f_{j}^{(n)} k_{j}^{(n-1)} \sin \left(\theta_{2 j-1}^{(n)}+\theta_{2 j}^{(n)}\right), \quad 0 \leq x \leq x_{j, \pi-\alpha_{j}^{(n)}}^{(n)}, \\
2 f_{j}^{(n)} k_{j}^{(n-1)} \sin \left(\theta_{2 j-1}^{(n)}+\theta_{2 j}^{(n)}\right), \quad x_{j, \pi-\alpha_{j}^{(n)}}^{(n)} \leq x \leq\left(f_{j}^{(n)}\right)^{-1} g_{n}, \\
-\min \left(\delta k_{j}^{(n)}, \frac{g_{n}}{2 x}\right) \frac{k_{j}^{(n-1)}}{4}\left(\sin 2 \theta_{2 j-1}^{(n)}+\sin 2 \theta_{2 j}^{(n)}\right), \quad x>\left(f_{j}^{(n)}\right)^{-1} g_{n} .
\end{array}\right.
$$

Here $\alpha_{j}^{(n)}$ is defined by $f_{j}^{(n)} \sin \alpha_{j}^{(n)}=\delta k_{j}^{(n)}$, and

$$
x_{j, \beta}^{(n)}=\min \left\{x: \delta \theta_{j}^{(n)}(x)=\beta\right\} .
$$

Notice that the value of $g_{n}$ is going to be the same for all $j$. Also, as before, we will set $\tilde{g}_{n}=\exp \left(g_{n}^{3 / 4}\right)$. The parameters $g_{n}, f_{j}^{(n)}$ and $\delta k_{j}^{(n)}$ are to be chosen at the $n$th step of the construction. The energies $k_{j}^{(n-1)}$ are given (from the previous step). At the $n$th step, each $k_{j}^{(n-1)}$ splits into two eigenvalues $k_{2 j-1}^{(n)}, k_{2 j}^{(n)}$; that is, the interval $\left(k_{2 j-1}^{(n)}, k_{2 j}^{(n)}\right)$ is centered at $k_{j}^{(n-1)}$ and has length $\delta k_{j}^{(n)}$. Throughout the paper, we will assume the relationship $f_{j}^{(n)}=\tilde{g}_{n} \delta k_{j}^{(n)}$. In the actual construction, $V^{(n)}(x)$ will be given by (2.7) only to the right of some value of $x=x_{n}$, and $R\left(x_{n}, k_{j}^{(n)}\right) \neq 1$. However in this section we will consider $V^{(n)}(x)$ defined by (2.7) on $[0, \infty)$ and we will assume that $R(0, k)=1$ and $\theta_{j}^{(n)}(0, k)=0$ for all $k$. Let us introduce one more parameter that we need, $a_{n}=\min _{j, j^{\prime}}\left|k_{j}^{(n-1)}-k_{j^{\prime}}^{(n-1)}\right|$ (essentially, in the construction process we will have $a_{n}=\min _{j}\left(\delta k_{j}^{(n-1)}\right)$ ). To keep notation compact, henceforth in this section we are going to omit the index " $n$ " for most variables, including $V$ (but with the exception of the $k$ 's, where members of two different steps participate explicitly in a construction).

Lemma 2.3 (Splitting Lemma). Let $V(x)$ be given by (2.7), and assume that

$$
4 \sum_{j} f_{j} k_{j}^{(n-1)}<\frac{1}{3} a, \quad g>>1,2^{n} g a^{-2} \sum_{j=1}^{2^{n-1}} f_{j}<<1, \quad \delta k_{j}<\frac{1}{12} a,
$$

for $j=1, \ldots, 2^{n-1}$. Then for any $l=1, \ldots, 2^{n-1}$,

$$
\left\|R\left(x, k_{2(l-1)+i}^{(n)}\right)\right\|_{L^{2}\left(\mathbb{R}^{+}\right)}^{2}=2 f_{l}^{-1}\left(1+O\left(2^{n} e^{g} a^{-2} \sum_{j=1}^{2^{n-1}} f_{j}, \tilde{g}^{-1 / 2} g\right)\right),
$$

where $i=1,2$.

Remark. The condition $g>>1$ means that $g$ needs to be greater than some universal constant, the value of which can be derived from the proof. The same interpretation applies to the other condition in (2.8) involving " $<<$ ". Notice also that the fourth condition basically follows from the first two and $\delta k_{j}=\tilde{g}^{-1} f_{j}$, but we state it separately for convenience.

We are going to fix $l=1, i=1$ and prove the result for this case; other $l, i$ are treated in exactly the same way. Write $V=V_{1}+W$, where $V_{1}=V_{1}^{(n)}$ and $W$ 
is the rest of the potential. Direct substitution of (2.7) into (2.2), (2.3) and some trigonometry leads to the following equations valid for $x<f_{1}^{-1} g$ :

$$
\left(\log R^{2}\left(x, k_{1}^{(n)}\right)\right)^{\prime}=-\frac{k_{1}^{(n-1)}}{k_{1}^{(n)}} \tilde{f}_{1}(x)\left(\cos \delta \theta_{1}-\cos \left(\theta_{2}+3 \theta_{1}\right)\right)+\frac{W(x)}{k_{1}^{(n)}} \sin 2 \theta_{1},
$$

$$
\begin{aligned}
\left(\delta \theta_{1}\right)^{\prime}= & \delta k_{1}\left(1-\frac{V(x)}{2 k_{1}^{(n)} k_{2}^{(n)}}\left(1+\cos 2 \theta_{2}\right)\right) \\
& +\left(\frac{k_{1}^{(n-1)}}{k_{1}^{(n)}} \tilde{f}_{1}(x)\left(1-\cos 2\left(\theta_{1}+\theta_{2}\right)\right)-\frac{W(x)}{k_{1}^{(n)}} \sin \left(\theta_{1}+\theta_{2}\right)\right) \sin \delta \theta_{1} ;
\end{aligned}
$$

here $\tilde{f}_{1}(x)= \pm f_{1}$ with a change from + to - at $x=x_{1, \pi-\alpha_{1}}$. For the rest of this section, we are also going to omit the index " 1 " in $x_{1, \beta}$ and write simply $x_{\beta}$ for $x$ such that $\delta \theta_{1}\left(x_{\beta}\right)=\beta$.

Remark. Equations (2.10) and (2.11) are fairly complicated, but not all terms are of equal importance. At the heart of the matter is the following simpler nonlinear dynamical system:

$$
\begin{gathered}
\left(\log R^{2}\right)^{\prime}=-\tilde{f}_{1}(x) \cos \delta \theta_{1}, \\
\left(\delta \theta_{1}\right)^{\prime}=\delta k_{1}+\tilde{f}_{1}(x) \sin \delta \theta_{1} .
\end{gathered}
$$

In some sense, we will show that the rest of the terms in (2.10), (2.11) produce only small corrections to the behavior of this system. It is quite instructive (and technically simple) to prove a version of the Splitting Lemma for the system (2.12), but to save space, we are going to treat directly the system (2.10), (2.11).

From (2.11) and $\delta \theta_{1}(0)=0$, we can write

$$
\delta \theta_{1}=\delta k_{1} \int_{0}^{x} e^{\int_{y}^{x} \xi(s) d s}(1+O(|V(y)|)) d y,
$$

where

$$
\xi(y)=\frac{k_{1}^{(n-1)}}{k_{1}^{(n)}}\left(\tilde{f}_{1}(y)\left(1-\cos 2\left(\theta_{1}+\theta_{2}\right)\right)-\frac{W(y)}{k_{1}^{(n-1)}} \sin \left(\theta_{1}+\theta_{2}\right)\right) \frac{\sin \delta \theta_{1}}{\delta \theta_{1}} .
$$

We need the following technical lemma.

Lemma 2.4. Under the assumptions of the Splitting Lemma, we have for $y<x \leq$ $f_{1}^{-1} g$,

$$
\int_{y}^{x} \xi(s) d s=\int_{y}^{x} \tilde{f}_{1}(s) \frac{\sin \delta \theta_{1}}{\delta \theta_{1}} d s\left(1+O\left(\delta k_{1}\right)\right)+O\left(\mathcal{E}_{n}\right),
$$

where $\mathcal{E}_{n}=2^{n}\left(\sum_{j} f_{j}\right) g a^{-2}$.

Proof. Let us consider one of the terms entering into $\xi$,

$$
\int_{y}^{x} V_{j}(s) \sin \left(\theta_{1}+\theta_{2}\right) \frac{\sin \delta \theta_{1}}{\delta \theta_{1}} d s
$$


where $2 \leq j \leq 2^{n-1}$ is arbitrary. Recall that $V_{j}(s)=\tilde{f}_{j}(s) \sin \left(\theta_{2 j-1}+\theta_{2 j}\right)$ for $x \leq\left(f_{j}\right)^{-1} g$, where $\tilde{f}_{j}= \pm f_{j}$ with only at most one jump (we do not assume a priori that $x_{j, \pi-\alpha_{j}}<\left(f_{j}\right)^{-1} g$, but will show this below). For $x>\left(f_{j}\right)^{-1} g$, $V_{j}(s)=\tilde{f}_{j}(s)\left(\sin \left(2 \theta_{2 j-1}\right)+\sin \left(2 \theta_{2 j}\right)\right)$, where $\left|\tilde{f}_{j}(s)\right| \leq\left(k_{j}^{(n-1)} g\right) /(2 s),\left|\tilde{f}_{j}^{\prime}(s)\right| \leq$ $\left(k_{j}^{(n-1)} g\right) /\left(2 s^{2}\right)$. Notice that by assumption (2.8) we have

$\left|\left(\theta_{2 j-1}+\theta_{2 j}-\theta_{1}-\theta_{2}\right)^{\prime}\right| \geq \frac{a}{2}, \quad\left|\left(2 \theta_{2 j-1}-\theta_{1}-\theta_{2}\right)^{\prime}\right| \geq \frac{a}{2}, \quad\left|\left(2 \theta_{2 j}-\theta_{1}-\theta_{2}\right)^{\prime}\right| \geq \frac{a}{2}$.

Substituting the expression for $V_{j}$ into (2.16) and using the formula for the product of sines, we reduce matters to estimation of integrals of the type

$$
I_{ \pm}(x, y)=\int_{y}^{x} \tilde{f}_{j} \cos \left(2 \theta_{2 j} \pm\left(\theta_{1}+\theta_{2}\right)\right) \frac{\sin \delta \theta_{1}}{\delta \theta_{1}} d s .
$$

Consider the "-" case, and integrate by parts integrating

$$
\cos \left(2 \theta_{2 j}-\left(\theta_{1}+\theta_{2}\right)\right)\left(2 \theta_{2 j}-\left(\theta_{1}+\theta_{2}\right)\right)^{\prime} .
$$

Observing that $\left|\theta_{j}^{\prime \prime}(s)\right| \leq C\left(|V(s)|+\left|V^{\prime}(s)\right|\right)$ for any $j,\left|\left(\delta \theta_{1}\right)^{\prime}(s)\right| \leq C\left(|V(s)|+\delta k_{1}\right)$ and using the inequality (2.17) for the derivative of the argument of cosine, we obtain

$$
\begin{array}{r}
\left|I_{-}(x, y)\right| \leq C a^{-1}\left(f_{j}+\int_{y}^{x}\left(\left|\tilde{f}_{j}^{\prime}(s)\right|+\left|\tilde{f}_{j}(s)\right|\left(|V(s)|+\delta k_{1}\right)\right.\right. \\
\left.\left.+\left|\tilde{f}_{j}(s)\right|\left(|V(s)|+\left|V^{\prime}(s)\right|\right) a^{-1}\right) d s\right),
\end{array}
$$

where $C$ is a universal constant. The discontinuities of $\tilde{f}_{j}$ are taken into account in the off-integral term. Since $|V(s)|,\left|V^{\prime}(s)\right| \leq C \sum_{l}\left|\tilde{f}_{l}(s)\right|, \delta k_{1}<f_{1}$ and $a \leq C$, we arrive at a bound valid for all $y<x \leq f_{1}^{-1} g$ :

$$
\left|I_{-}(x, y)\right| \leq C\left(f_{j} a^{-1}+a^{-2} \int_{y}^{x}\left|\tilde{f}_{j}(s)\right| \sum_{l}\left|\tilde{f}_{l}(s)\right| d s\right) .
$$

The estimate on $I_{+}$is similar, but involves a constant independent of $n$ instead of $a$ (arising from the minimal possible size of $k_{j}^{(n)}$ ). The contribution of the term $\tilde{f}_{1}(s) \cos \left(2\left(\theta_{1}+\theta_{2}\right)\right)$ in (2.14) is bounded similarly to $I_{+}$. Summing up all bounds, we obtain at most

$$
C\left(\sum_{j} f_{j} a^{-1}+a^{-2} \int_{y}^{x}\left(\sum_{j}\left|\tilde{f}_{j}(s)\right|\right)^{2} d s\right) .
$$

We can compute explicitly that $\left\|\tilde{f}_{j}\right\|_{L^{2}}^{2} \leq 2 f_{j} g$, and therefore the total bound does not exceed $C a^{-2} 2^{n}\left(\sum_{j} f_{j}\right) g$. Notice that the first term in (2.18) is included into this error since $a$ is less than a fixed constant and $g>1$. This is exactly the error term in (2.15); the origin of the main term is clear. 
Remark. The error $O\left(\delta k_{1}\right)$ in the main term of (2.15) gives after integration $O\left(g \delta k_{1}\right)=O\left(\tilde{g}^{-1} g f_{1}\right)$, which can also be subsumed into $\mathcal{E}_{n}$.

Next we show that, provided that the error terms in (2.15) are small, we have $x_{\pi-\alpha_{1}}<f_{1}^{-1} g$, and thus our potential is well defined.

Lemma 2.5. For sufficiently large $g$ and for sufficiently small $\mathcal{E}_{n}$, we have

$$
x_{\pi-\alpha_{1}} \leq \pi f_{1}^{-1} \log (2 \tilde{g})\left(1+C \mathcal{E}_{n}\right) .
$$

Remark. Similarly to Lemma 2.3 by sufficiently large (or small) we mean that there exist universal constants such that if the value of $g$ (respectively $\mathcal{E}_{n}$ ) exceeds (is less than) these constants, the result holds.

Proof. Let us first estimate from above $x_{\pi / 2}$. On the interval $\left[0, x_{\pi / 2}\right]$ we have

$$
2 / \pi \leq\left(\sin \delta \theta_{1}\right) / \delta \theta_{1} \leq 1
$$

Thus, according to (2.13), Lemma 2.4 and the remark after its proof, we have

$$
\delta \theta_{1}(x) \geq e^{\frac{2}{\pi} f_{1} x} \frac{\pi \delta k_{1}}{2 f_{1}}\left(1-e^{-\frac{2}{\pi} f_{1} x}\right)\left(1-C \mathcal{E}_{n}\right)
$$

for $x<\left(f_{1}\right)^{-1} g$ and such that $\delta \theta_{1} \in[0, \pi / 2]$. It is clear from 2.20) that if $g$ is large enough, then $x_{\pi / 2}<f_{1}^{-1} g$. Indeed, recall that $\delta k_{1} / f_{1}=e^{-g^{3 / 4}}$. If $f_{1}^{-1} g$ were less than $x_{\pi / 2}$, 2.20) would imply that

$$
\delta \theta_{1}\left(f_{1}^{-1} g\right) \geq e^{\frac{2}{\pi} g-g^{3 / 4}} \frac{\pi}{2}\left(1-e^{-\frac{2}{\pi} g}\right)\left(1-C \mathcal{E}_{n}\right),
$$

an obvious contradiction for $g$ large enough and sufficiently small $\mathcal{E}_{n}$. Moreover, provided that $\mathcal{E}_{n}$ is small, we can estimate (assuming also $x_{\pi / 2} \geq \frac{\pi}{2}\left(f_{1}\right)^{-1} \log 2$, or else we have a nice upper bound)

$$
e^{\frac{2}{\pi} f_{1} x_{\pi / 2}} \leq 2 \tilde{g}\left(1+C \mathcal{E}_{n}\right) .
$$

From this inequality it is easy to see that

$$
x_{\pi / 2} \leq \frac{\pi}{2} f_{1}^{-1} \log (2 \tilde{g})\left(1+C \mathcal{E}_{n}\right) .
$$

Imagine for a moment that $\tilde{f}_{1}(x)=f_{1}$ for $x \leq x_{\pi}$. Notice that under a change of variables $\overline{\delta \theta}_{1}=\pi-\delta \theta_{1}, \bar{x}=x_{\pi}-x$ the equation (2.11) transforms into the equation for $\overline{\delta \theta}_{1}$ of the same form as (2.11), with the same initial condition and the same estimates available. In particular, the point $\bar{x}_{\pi / 2}$ such that $\overline{\delta \theta}_{1}\left(\bar{x}_{\pi / 2}\right)=\pi / 2$ satisfies the same bound as $x_{\pi / 2}$. But $\overline{\delta \theta}_{1}(\bar{x})=\pi-\delta \theta_{1}\left(x_{\pi}-x\right)$, and thus $\bar{x}_{\pi / 2}=x_{\pi}-x_{\pi / 2}$. But $x_{\pi-\alpha_{1}} \leq x_{\pi}$, and the above observation together with (2.21) finishes the proof of the lemma.

Next, we prove the following essential

Lemma 2.6 (Small Angle Lemma). Let $\gamma$ be a small angle, in particular such that $\gamma<g^{-1}$. Denote by $x_{\gamma}$ the smallest value of $x$ where $\delta \theta_{1}(x)=\gamma$. Then

$$
R\left(x_{\gamma}, k_{1}^{(n)}\right)^{2}=\frac{\delta k_{1}}{\gamma f_{1}+\delta k_{1}}\left(1+O\left(\gamma^{2} g, \mathcal{E}_{n}\right)\right) .
$$

Moreover,

$$
\left\|R\left(x, k_{1}^{(n)}\right)\right\|_{L^{2}\left(0, x_{\gamma}\right)}^{2}=f_{1}^{-1} \frac{1}{1+\tilde{g}^{-1} \gamma^{-1}}\left(1+O\left(\gamma^{2} g, \mathcal{E}_{n}\right)\right) .
$$


Proof. Notice that on $\left[0, x_{\gamma}\right]$, we have $\left(\sin \delta \theta_{1}\right) / \delta \theta_{1}=1+O\left(\gamma^{2}\right)$. Observe that by Lemma 2.5 we have that $x_{\gamma}<f_{1}^{-1} g$. By 2.13), Lemma 2.4 and the definition of potential we have that

$$
\delta k_{1} \int_{0}^{x_{\gamma}} e^{f_{1}\left(x_{\gamma}-y\right)\left(1+O\left(\gamma^{2}\right)\right)+O\left(\mathcal{E}_{n}\right)}(1+O(|V(y)|) d y=\gamma .
$$

Thus, we obtain

$$
e^{f_{1} x_{\gamma}}\left(1-e^{-f_{1} x_{\gamma}}\right) f_{1}^{-1} \delta k_{1}\left(1+O\left(\mathcal{E}_{n}, \gamma^{2} g\right)\right)=\gamma
$$

We included the $O(|V(y)|)$ error into $\mathcal{E}_{n}$, since we can assume freely that $a<1$ and $g>1$. Thus

$$
e^{f_{1} x_{\gamma}}=\left(\frac{\gamma f_{1}}{\delta k_{1}}+1\right)\left(1+O\left(\gamma^{2} g, \mathcal{E}_{n}\right)\right) .
$$

Now in the equation (2.10) for the amplitude, we have for $x<f_{1}^{-1} g$,

$$
\log \left(R\left(x, k_{1}^{(n)}\right)^{2}\right)=-\int_{0}^{x} \tilde{f}_{1}(y) \cos \delta \theta_{1}(y) d y+O\left(\mathcal{E}_{n}\right),
$$

by an estimate directly analogous to the estimates of Lemma 2.4 (but easier). Therefore, for $x \leq x_{\gamma}$, we have

$$
R\left(x, k_{1}^{(n)}\right)^{2}=e^{-f_{1} x}\left(1+O\left(\gamma^{2} g, \mathcal{E}_{n}\right)\right) .
$$

The first statement of the lemma, estimate (2.22), follows directly from (2.27) and (2.25). Also, the $L^{2}$ norm of $R\left(x, k_{1}^{(n)}\right)$ on $\left[0, x_{\gamma}\right]$ satisfies

$$
\begin{aligned}
\left\|R\left(x, k_{1}^{(n)}\right)\right\|_{L^{2}\left(0, x_{\gamma}\right)}^{2} & =f_{1}^{-1}\left(1-e^{-f_{1} x_{\gamma}}\right)\left(1+O\left(\gamma^{2} g, \mathcal{E}_{n}\right)\right) \\
& =f_{1}^{-1} \frac{1}{1+\tilde{g}^{-1} \gamma^{-1}}\left(1+O\left(\gamma^{2} g, \mathcal{E}_{n}\right)\right) .
\end{aligned}
$$

In the last step we used (2.22) and the fact that $\delta k_{1}=\tilde{g}^{-1} f_{1}$.

Next we need a lemma describing the solution of (2.10), 2.11) on the interval $\left[0, x_{\pi-\alpha_{1}}\right]$. Let $R\left(x, k_{1}^{(n)}\right), \delta \theta_{1}$ solve (2.10), (2.11) and assume that $\tilde{R}(x), \tilde{\delta} \theta_{1}(x)$ solve a variant of a system (2.12)

$$
\begin{aligned}
\left(\log \tilde{R}^{2}(x)\right)^{\prime} & =-f_{1} \cos \tilde{\delta \theta_{1}}(x) \\
\left(\tilde{\delta}_{1}(x)\right)^{\prime} & =\delta k_{1}+f_{1} \sin \tilde{\delta \theta_{1}}(x)
\end{aligned}
$$

for all $x$. Assume furthermore that the initial conditions for $\tilde{R}, \tilde{\delta \theta} \theta_{1}$ at $x=0$ are the same as for $R, \delta \theta$. Then the following lemma holds.

Lemma 2.7. For every $x \leq x_{\pi-\alpha_{1}}$, we have

$$
\begin{array}{r}
\left|\tilde{\delta} \theta_{1}(x)-\delta \theta_{1}(x)\right| \leq C \tilde{g}^{-1} \tilde{\mathcal{E}}_{n}, \\
\tilde{R}^{2}(x) / R^{2}\left(x, k_{1}^{(n)}\right)=1+O\left(\tilde{\mathcal{E}}_{n}\right),
\end{array}
$$

where $\tilde{\mathcal{E}}_{n}=2^{n} e^{g} a^{-2} \sum_{j} f_{j}$. 
Proof. Define $\delta \theta^{*}(x)=\tilde{\delta} \theta_{1}(x)-\delta \theta_{1}(x)$. From (2.11), (2.29) it follows that for $x \leq f_{1}^{-1} g$,

$$
\begin{aligned}
\left(\delta \theta^{*}\right)^{\prime}(x)= & \delta k_{1} O(|V(x)|)+f_{1} \frac{\sin \tilde{\delta \theta_{1}}-\sin \delta \theta_{1}}{\delta \theta^{*}} \delta \theta^{*} \\
& +\left(f_{1} \cos 2\left(\theta_{1}+\theta_{2}\right)+\frac{W(x)}{k_{1}^{(n)}} \sin \left(\theta_{1}+\theta_{2}\right)\right) \sin \delta \theta_{1} .
\end{aligned}
$$

Therefore,

$$
\begin{aligned}
\delta \theta^{*}(x)=\int_{0}^{x} e^{\int_{y}^{x} \eta(s) d s}( & \delta k_{1} O(|V(y)|) \\
& \left.+\left(f_{1} \cos 2\left(\theta_{1}+\theta_{2}\right)+\frac{W(x)}{k_{1}^{(n)}} \sin \left(\theta_{1}+\theta_{2}\right)\right) \sin \delta \theta_{1}\right),
\end{aligned}
$$

where

$$
\eta(s)=2 f_{1} \frac{\sin \delta \theta^{*}}{\delta \theta^{*}} \cos \left(\delta \theta_{1}+\tilde{\delta}_{1}\right) .
$$

Now all oscillatory terms in (2.32) (that is, all terms except the $\delta k_{1} O(|V|)$ term) are estimated by integration by parts similarly to Lemma 2.4. For example, for any of the $2^{n-1}-1$ terms entering into $W(y)$, we have for $x \leq x_{\pi-\alpha_{1}}$,

$$
\begin{aligned}
& \left|\int_{0}^{x} e^{\int_{y}^{x} \eta(s) d s} V_{j}(y) \sin \left(\theta_{1}+\theta_{2}\right) d y\right| \\
& \quad \leq C a^{-1} \tilde{g}\left(f_{j}+\int_{0}^{x}\left(\left|\tilde{f}_{j}^{\prime}(y)\right|+|\eta(y)|\left|\tilde{f}_{j}(y)\right|+\left|\tilde{f}_{j}(y)\right| O\left(|V(y)|+\left|V^{\prime}(y)\right|\right) a^{-1}\right) d y\right),
\end{aligned}
$$

where

$$
\tilde{f}_{j}(y)= \begin{cases}-f_{j}, & 0 \leq x \leq x_{j, \pi-\alpha_{j}} \\ f_{j}, & x_{j, \pi-\alpha_{j}} \leq x \leq\left(f_{j}\right)^{-1} g, \\ -\min \left(\delta k_{j}, \frac{g}{2 x}\right) \frac{k_{j}^{(n-1)}}{4}, & x>\left(f_{j}\right)^{-1} g .\end{cases}
$$

Lemma 2.5 was used to estimate the exponential by $\tilde{g}$. We make a convention that the derivative $\tilde{f}_{j}^{\prime}(y)$ does not involve $\delta$ functions from the two jumps of $\tilde{f}_{j}$; those jumps contribute to the first, off-integral term in the estimate. Notice that $|\eta(x)|,|V(x)|,\left|V^{\prime}(x)\right| \leq C \sum_{j} \tilde{f}_{j}(x)$, where $C$ is a universal constant. Thus the total bound in (2.33) does not exceed

$$
C a^{-1} \tilde{g}\left(f_{j}+a^{-1} \int_{0}^{\infty} \tilde{f}_{j}(y) \sum_{l} \tilde{f}_{l}(y) d y\right) .
$$

The term $f_{1} \cos 2\left(\theta_{1}+\theta_{2}\right)$ is estimated similarly (but gives a smaller error), while the term $\delta k_{1} O(|V(y)|)$ integrated over $\left[0, x_{\pi-\alpha_{1}}\right]$ with the exponential gives at most 
$\left(f_{1}\right)^{-1} \delta k_{1} \tilde{g} \sum_{j} f_{j}$ by Lemma 2.5 Summing together all errors we obtain

$$
\left|\delta \theta^{*}\right| \leq C \tilde{g}\left(a^{-1} \sum_{l} f_{l}+a^{-2} \int_{0}^{\infty}\left(\sum_{j} \tilde{f}_{j}\right)^{2} d y\right),
$$

which leads to

$$
\left|\delta \theta^{*}(x)\right| \leq C 2^{n} \tilde{g} g a^{-2} \sum_{j} f_{j}
$$

for $x \leq x_{\pi-\alpha_{1}}$. This proves (2.30), since $e^{g} \geq C g\left(\tilde{g}^{2}\right)$ for some universal $C$ and all $g$.

By (2.10), (2.28) and an argument parallel to Lemma 2.4,

$$
\log R^{2}\left(x, k_{1}^{(n)}\right)-\log \tilde{R}^{2}(x)=-\int_{0}^{x} f_{1}\left(\cos \delta \theta_{1}-\cos \tilde{\delta} \theta_{1}\right) d y+O\left(\mathcal{E}_{n}\right) .
$$

Using (2.30) and Lemma 2.5] we obtain for $x \leq x_{\pi-\alpha_{1}}$,

$$
R^{2}\left(x, k_{1}^{(n)}\right) / \tilde{R}^{2}(x)=1+O\left(\tilde{\mathcal{E}_{n}}\right),
$$

which finishes the proof (we used that evidently $\mathcal{E}_{n}<\tilde{\mathcal{E}_{n}}$ and so the former error is absorbed by the latter). Also, at the last step we generously exchanged $\tilde{g}^{-1}$ for $g^{3 / 4}$ to arrive at an error in (2.36).

Now we are ready to proceed with the proof of Lemma 2.3

Proof of the Splitting Lemma. The structure of the eigenfunctions obtained in the Splitting Lemma is illustrated by Figure 1 .

1. Consider first the interval $\left[0, x_{\pi / 2}\right]$. The estimate for the norm of $R\left(x, k_{1}^{(n)}\right)$ on the interval $\left[0, x_{\gamma}\right]$ for some small $\gamma$ is provided by Lemma 2.6 By (2.22) and (2.26), the norm on the interval $\left[x_{\gamma}, x_{\pi / 2}\right]$ does not exceed $\left(\frac{\gamma f_{1}}{\delta k_{1}}+1\right)^{-1} x_{\pi / 2}\left(1+O\left(\gamma^{2} g, \mathcal{E}_{n}\right)\right)$. Choosing $\gamma=\tilde{g}^{-1 / 2}$, for example, and using the bound (2.21) on $x_{\pi / 2}$, we find that

$$
\left\|R\left(x, k_{1}^{(n)}\right)\right\|_{L^{2}\left[x_{\gamma}, x_{\pi / 2}\right]}^{2}=f_{1}^{-1} O\left(\tilde{g}^{-1 / 2} g^{3 / 4}, \mathcal{E}_{n}\right) .
$$

Thus, altogether,

$$
\left\|R\left(x, k_{1}^{(n)}\right)\right\|_{L^{2}\left[0, x_{\pi / 2}\right]}^{2}=f_{1}^{-1}\left(1+O\left(\mathcal{E}_{n}, \tilde{g}^{-1 / 2} g\right)\right) .
$$

2. Next, we estimate the norm on $\left[x_{\pi / 2}, x_{\pi-\alpha_{1}}\right]$. Consider an auxiliary simplified system (2.28), 2.29) of Lemma 2.7. By (2.30), we have

$$
\tilde{\delta \theta_{1}}\left(x_{\pi / 2}\right)=\pi / 2+O\left(\tilde{g}^{-1} \tilde{\mathcal{E}}_{n}\right), \quad \tilde{\delta \theta}_{1}\left(x_{\pi-\alpha_{1}}\right)=\pi-\alpha_{1}+O\left(\tilde{g}^{-1} \tilde{\mathcal{E}}_{n}\right) .
$$

Define $\tilde{x}_{\beta}=\min \left\{x \mid \tilde{\delta \theta_{1}}(x)=\beta\right\}$. Notice that the system (2.28), (2.29) is symmetric in the sense that for $x \leq \tilde{x}_{\pi}$,

$$
\tilde{\delta \theta}_{1}(x)=\pi-\tilde{\delta \theta_{1}}\left(\tilde{x}_{\pi}-x\right) \text { and } \tilde{R}^{2}(x)=\tilde{R}^{2}\left(\tilde{x}_{\pi}-x\right) .
$$

Observe also that $\alpha_{1}=\tilde{g}_{1}^{-1}\left(1+O\left(\tilde{g}^{-2}\right)\right)$. Thus the norm

$$
\left.\|\tilde{R}\|_{L^{2}\left[x_{\pi / 2}, x_{\pi-\alpha_{1}}\right]}^{2}=\|\tilde{R}\|_{L^{2}\left[\tilde{x}_{\alpha_{1}\left(1+O\left(\tilde{\varepsilon}_{n}\right)\right)}\right.}^{2} \tilde{x}_{\pi / 2\left(1+O\left(\tilde{\varepsilon}_{n}\right)\right)}\right] .
$$

By identical arguments, Lemma 2.5 and Lemma 2.6 (Small Angle Lemma) apply to the system (2.28), (2.29) (with the only difference that the conclusion does not involve the error $\mathcal{E}_{n}$ coming from oscillatory terms which are absent in the 


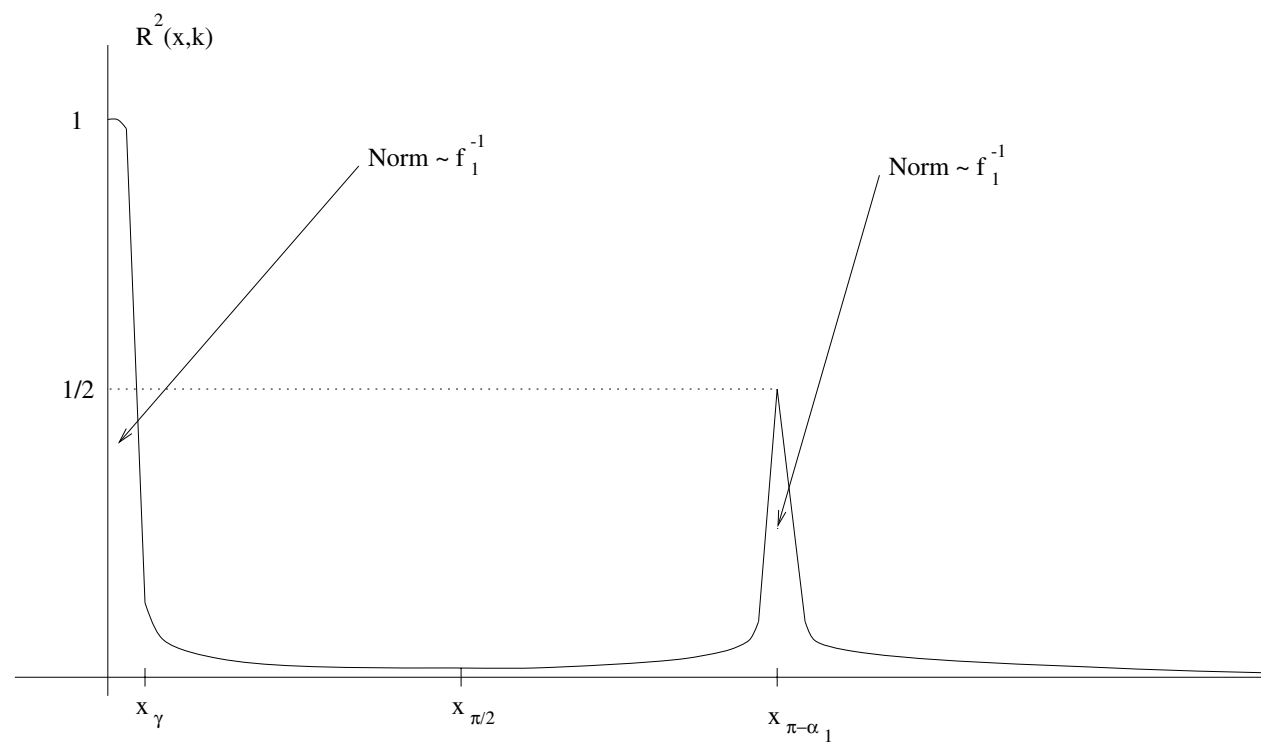

Figure 1. The structure of the eigenfunction at $k=k_{1,2}^{(n)}$. The error terms are not pictured and lead to small oscillations around the main value.

simplified system). Therefore, the considerations close to the first part of the proof above apply to the norm of $\tilde{R}$ and give

$$
\|\tilde{R}\|_{L^{2}\left[0, \tilde{x}_{\pi / 2\left(1+O\left(\tilde{\mathcal{E}}_{n}\right)\right)}^{2}\right]}^{2}=f_{1}^{-1}\left(1+O\left(\tilde{g}^{-1 / 2} g\right)\right) .
$$

By (2.23) of Lemma 2.6, we also have

$$
\|\tilde{R}\|_{L^{2}\left[0, \tilde{x}_{\alpha_{1}\left(1+O\left(\tilde{\varepsilon}_{n}\right)\right)}\right.}^{2}=\frac{1}{2} f_{1}^{-1}\left(1+O\left(\tilde{\mathcal{E}}_{n}, \tilde{g}^{-2} g\right)\right) .
$$

Therefore, the norm in (2.38) is equal to $\frac{1}{2} f_{1}^{-1}\left(1+O\left(\tilde{\mathcal{E}}_{n}, \tilde{g}^{-1 / 2} g\right)\right)$. Now by the estimate (2.31) of Lemma 2.7 we have

$$
\left\|R\left(x, k_{1}^{(n)}\right)\right\|_{L^{2}\left[x_{\pi / 2}, x_{\pi-\alpha_{1}}\right]}^{2}=\frac{1}{2} f_{1}^{-1}\left(1+O\left(\tilde{\mathcal{E}}_{n}, \tilde{g}^{-1 / 2} g\right)\right) .
$$

In addition, by Lemma 2.6 and symmetry

$$
\tilde{R}^{2}\left(x_{\pi-\alpha_{1}}\right)=\tilde{R}^{2}\left(x_{\alpha_{1}\left(1+O\left(\tilde{\mathcal{E}_{n}}\right)\right)}\right)=\frac{1}{2}\left(1+O\left(\tilde{\mathcal{E}}_{n}, \tilde{g}^{-2} g\right)\right) .
$$

By Lemma 2.7 this implies

$$
R\left(x_{\pi-\alpha_{1}}, k_{1}^{(n)}\right)^{2}=\frac{1}{2}\left(1+O\left(\tilde{\mathcal{E}}_{n}, \tilde{g}^{-2} g\right)\right) .
$$

3. The next step is the consideration of the $\left[x_{\pi-\alpha_{1}}, f_{1}^{-1} g\right]$ interval. On this interval, in the idealized system (2.12) the angle $\delta \theta_{1}$ stays constant, and since it is very close to $\pi$, this allows for a consistent decay in the $R$ equation. The main goal therefore is to control the additional perturbation terms, which are either small or 
oscillating. Define $\delta \bar{\theta}_{1}=\left(\pi-\alpha_{1}\right)-\delta \theta_{1}$. Then

$$
\begin{aligned}
\left(\delta \bar{\theta}_{1}\right)^{\prime}= & -\delta k_{1}(1+O(|V(x)|)) \\
& +\sin \left(\delta \bar{\theta}_{1}+\alpha_{1}\right)\left(f_{1}\left(1-\cos 2\left(\theta_{1}+\theta_{2}\right)\right)+\frac{W(x)}{k_{1}^{(n)}} \sin \left(\theta_{1}+\theta_{2}\right)\right) .
\end{aligned}
$$

Using the relationship $\delta k_{1}=f_{1} \sin \alpha_{1}$, we arrive at

$$
\begin{aligned}
\left(\delta \bar{\theta}_{1}\right)^{\prime}= & \left(f_{1}\left(1-\cos 2\left(\theta_{1}+\theta_{2}\right)\right)+\frac{W(x)}{k_{1}^{(n)}} \sin \left(\theta_{1}+\theta_{2}\right)\right) \\
& \times\left(\cos \alpha_{1} \frac{\sin \delta \bar{\theta}_{1}}{\delta \bar{\theta}_{1}}+\sin \alpha_{1} \frac{\cos \delta \bar{\theta}_{1}-1}{\delta \bar{\theta}_{1}}\right) \delta \bar{\theta}_{1} \\
& -\delta k_{1} O(|V(x)|)+f_{1} \sin \alpha_{1} \cos 2\left(\theta_{1}+\theta_{2}\right)-\frac{W(x)}{k_{1}^{(n)}} \sin \alpha_{1} \sin \left(\theta_{1}+\theta_{2}\right),
\end{aligned}
$$

with the initial data $\delta \bar{\theta}_{1}\left(x_{\pi-\alpha_{1}}\right)=0$. Denote by $\zeta(x)$ the expression in front of $\delta \bar{\theta}_{1}$ in (2.43). The solution to (2.43) can be written in the following form:

$$
\delta \bar{\theta}_{1}(x)=\delta k_{1} \int_{x_{\pi-\alpha_{1}}}^{x} e^{\int_{y}^{x} \zeta(s) d s}\left(O(|V(y)|)-\cos 2\left(\theta_{1}+\theta_{2}\right)-\frac{W(y)}{f_{1} k_{1}^{(n)}} \sin \left(\theta_{1}+\theta_{2}\right)\right) d y .
$$

Notice that for $x_{\pi-\alpha_{1}} \leq y \leq x \leq\left(f_{1}\right)^{-1} g$,

$$
\int_{y}^{x} \zeta(s) d s=f_{1} \int_{y}^{x}\left(\frac{\sin \delta \bar{\theta}_{1}}{\delta \bar{\theta}_{1}} \cos \alpha_{1}+\frac{\cos \delta \bar{\theta}_{1}-1}{\delta \bar{\theta}_{1}} \sin \alpha_{1}\right) d s+O\left(\mathcal{E}_{n}\right)
$$

by the same argument as in the proof of Lemma 2.4. Therefore, since $\alpha_{1} \sim \tilde{g}^{-1}$,

$$
e^{\int_{y}^{x} \zeta(s) d s} \leq e^{f_{1}(x-y)}\left(1+O\left(\tilde{g}^{-1} g, \mathcal{E}_{n}\right)\right)
$$

for $x \leq\left(f_{1}\right)^{-1} g$. All oscillatory terms in (2.44) (that is, all terms except the $O(|V|)$ term) are estimated by integration by parts similarly to Lemma 2.4 and Lemma 2.7 For example, for any of the $2^{n-1}-1$ terms entering into $W(y)$, we have for $x_{\pi-\alpha_{1}} \leq$ $x \leq f_{1}^{-1} g$,

$$
\begin{aligned}
& \left|\int_{x_{\pi-\alpha_{1}}}^{x} e^{\int_{y}^{x} \zeta(s) d s} V_{j}(y) \sin \left(\theta_{1}+\theta_{2}\right) d y\right| \\
& \leq C a^{-1} e^{g}\left(f_{j}+\int_{x_{\pi-\alpha_{1}}}^{\infty}\left(\left|\tilde{f}_{j}^{\prime}(y)\right|+|\zeta(y)|\left|\tilde{f}_{j}(y)\right|+\left|\tilde{f}_{j}(y)\right| O\left(|V(y)|+\left|V^{\prime}(y)\right|\right) a^{-1}\right) d y\right),
\end{aligned}
$$

where $\tilde{f}_{j}$ is given by 2.34 as before. Similarly to the computation in Lemma 2.7 the total bound in (2.47) is

$$
C e^{g}\left(f_{j} a^{-1}+a^{-2} \int_{x_{\pi-\alpha_{1}}}^{\infty} \tilde{f}_{j}(y) \sum_{l} \tilde{f}_{l}(y) d y\right)
$$


The term $\delta k_{1} \cos 2\left(\theta_{1}+\theta_{2}\right)$ is estimated similarly (but gives a smaller error), while the term $\delta k_{1} O(|V(y)|)$ integrated over $\left[x_{\pi-\alpha_{1}}, f_{1}^{-1} g\right]$ with the exponential gives at most $\left(f_{1}\right)^{-1} \delta k_{1} e^{g} \sum_{j} f_{j}$. Summing together all errors we obtain

$$
|\delta \bar{\theta}(x)| \leq C 2^{n} \tilde{g}^{-1} g e^{g} a^{-2} \sum_{j} f_{j} .
$$

Now notice that

$\left(\log R\left(x, k_{1}^{(n)}\right)^{2}\right)^{\prime}=\frac{k_{1}^{(n-1)}}{k_{1}^{(n)}} f_{1}\left(-\cos \left(\delta \bar{\theta}_{1}+\alpha_{1}\right)-\cos \left(\theta_{2}+3 \theta_{1}\right)\right)+\frac{W(x)}{k_{1}^{(n)}} \sin 2 \theta\left(x, k_{1}^{(n)}\right)$.

Using (2.42) and estimates parallel to the ones considered in Lemma 2.4 we find for $x_{\pi-\alpha_{1}} \leq x \leq\left(f_{1}\right)^{-1} g$ that

$$
R^{2}\left(x, k_{1}^{(n)}\right)=\frac{1}{2} e^{-f_{1}\left(x-x_{\pi-\alpha_{1}}\right)}\left(1+O\left(\tilde{\mathcal{E}}_{n}, g \tilde{g}^{-1}\right)\right),
$$

where as before $\tilde{\mathcal{E}}_{n}=2^{n} e^{g} a^{-2} \sum_{j} f_{j}$ (we are being generous here, replacing $g^{2} \tilde{g}^{-1} \tilde{\mathcal{E}}_{n}$ $+\mathcal{E}_{n}$ by $\tilde{\mathcal{E}}_{n}$ ). We also absorbed the error $\mathcal{E}_{n}$, coming from estimates of oscillatory integrals parallel to Lemma 2.4, into $\tilde{\mathcal{E}}_{n}$. In particular,

$$
\left\|R\left(x, k_{1}^{(n)}\right)\right\|_{L^{2}\left[x_{\pi-\alpha_{1}},\left(f_{1}\right)^{-1} g\right]}^{2}=\frac{1}{2} f_{1}^{-1}\left(1+O\left(\tilde{\mathcal{E}}_{n}, g \tilde{g}^{-1}\right)\right)
$$

and, given the upper bound on $x_{\pi-\alpha_{1}}$ of Lemma 2.5,

$$
R^{2}\left(f_{1}^{-1} g, k_{1}^{(n)}\right) \leq e^{-g / 2}\left(1+C\left(\tilde{\mathcal{E}}_{n}+\tilde{g}^{-1} g\right)\right) .
$$

4. Finally, we consider the solution on $\left[f_{1}^{-1} g, \infty\right)$. Here,

$$
V_{1}(x)=-\min \left(\delta k_{1}, \frac{g}{2 x}\right) \frac{k_{1}^{(n-1)}}{4}\left(\sin 2 \theta_{1}+\sin 2 \theta_{2}\right) .
$$

Equations for $\delta \theta_{1}$ and $R\left(x, k_{1}^{(n)}\right)$ can be written in the following form:

$$
\begin{aligned}
\left(\log R^{2}\left(x, k_{1}^{(n)}\right)\right)^{\prime}= & -\frac{k_{1}^{(n-1)}}{8 k_{1}^{(n)}} \min \left(\delta k_{1}, \frac{g}{2 x}\right)\left(1+\cos 2 \delta \theta_{1}-\cos 4 \theta_{1}-\cos 2\left(\theta_{1}+\theta_{2}\right)\right) \\
& +\frac{W(x)}{k_{1}^{(n)}} \sin 2 \theta_{1},
\end{aligned}
$$

$$
\begin{aligned}
\left(\delta \theta_{1}\right)^{\prime}=\delta k_{1}(1 & \left.+O\left(\delta k_{1}\right)\right)+\frac{k_{1}^{(n-1)}}{8 k_{1}^{(n)}} \min \left(\delta k_{1}, \frac{g}{2 x}\right) \sin 2 \delta \theta_{1}\left(1-\cos 2\left(\theta_{1}+\theta_{2}\right)\right) \\
+ & \frac{W(x)}{2 k_{1}^{(n)}}\left(\cos 2 \theta_{2}-\cos 2 \theta_{1}\right) .
\end{aligned}
$$

First of all, any contribution to the solution $R$ of the equation (2.51) coming from the last term involving $W$ or the last two summands in parentheses in the first term is of the order $O\left(\mathcal{E}_{n}\right)$ by the same estimates as before (in Lemma 2.4). Therefore,

$$
\frac{R\left(x, k_{1}^{(n)}\right)^{2}}{R\left(f_{1}^{-1} g, k_{1}^{(n)}\right)^{2}}=e^{-\frac{1}{8} \min \left(\delta k_{1}, \frac{g}{2 x}\right)\left(1+O\left(\delta k_{1}\right)\right) \int_{f_{1}^{-1} g}^{x}\left(1+\cos 2 \delta \theta_{1}\right) d y}\left(1+O\left(\mathcal{E}_{n}\right)\right) .
$$


Furthermore, the same estimates show that for any $x>f_{1}^{-1} g$,

$$
\int_{f_{1}^{-1} g}^{x} W(y)\left(\cos 2 \theta_{2}-\cos 2 \theta_{1}\right) d y=O\left(\mathcal{E}_{n}\right)
$$

as well. Consider a sequence of points $y_{n}$, where $y_{1}=f_{1}^{-1} g$, and

$$
y_{n}=\min \left\{y: \delta \theta_{1}(y)=\pi n+\delta \theta_{1}\left(y_{1}\right)\right\} .
$$

According to (2.52), (2.54) and (2.6), we have $y_{n}-y_{n-1} \leq\left(2 \pi+O\left(\mathcal{E}_{n}\right)\right)\left(\delta k_{1}\right)^{-1}$, and in each $\left(y_{n-1}, y_{n}\right)$ there is an interval $I_{n}$ of length at least $\left(\pi / 4+O\left(\mathcal{E}_{n}\right)\right)\left(\delta k_{1}\right)^{-1}$ where $\cos 2 \delta \theta_{1}>0$. Let us assume for simplicity that the error terms in the above estimates do not consume more than half of the principal terms. Then from (2.51) we see that on $\left(y_{n}, y_{n+1}\right)$,

$$
R\left(y, k_{1}^{(n)}\right)^{2} \leq C R\left(y_{1}, k_{1}^{(n)}\right)^{2} e^{-\sum_{j=1}^{n-1} b_{j}},
$$

where

$$
b_{n}=\frac{1}{16} \int_{I_{n}} \min \left(\delta k_{1}, \frac{g}{2 x}\right) d x \geq C \min \left(1, \frac{g}{g \tilde{g}^{-1}+3 \pi n}\right) .
$$

Taking into account (2.50) and the estimates on $b_{n}, y_{n}$ we have

$$
\left\|R\left(y, k_{1}^{(n)}\right)\right\|_{L^{2}\left[f_{1}^{-1} g, \infty\right)}^{2} \leq C e^{-g / 2} \sum_{n=0}^{\infty} e^{-\sum_{j=1}^{n} b_{j}}\left(\delta k_{1}\right)^{-1} .
$$

Recall that $\delta k_{1}=f_{1}^{-1} e^{g^{3 / 4}}$. Provided that $g$ is large enough so that the sum in (2.57) is finite, we can write

$$
\left\|R\left(y, k_{1}^{(n)}\right)\right\|_{L^{2}\left[f_{1}^{-1} g, \infty\right)}^{2}=f_{1}^{-1} O\left(e^{-g / 6}\right) .
$$

Combining the estimates (2.37), (2.41), (2.49) and (2.58), we get the Splitting Lemma.

\section{A CONTINUity LEMma}

In this section, we prove an auxiliary technical lemma that we are going to need in the construction. The estimates here are fairly straightforward, if technical. Moreover, we choose simplicity over sophistication and prove a version of the result which is sufficient for the application we have in mind. With more extensive technical effort, the estimates in this section can be significantly improved.

Assume that the potential $V^{(n)}(x)$ is given on an interval $\left(x_{n}, \infty\right)$ by $V^{(n)}(x)=$ $\sum_{j=1}^{2^{n-1}} V_{j}^{(n)}(x)$, where similarly to (2.7) we have

$$
V_{j}^{(n)}(x)=\left\{\begin{array}{l}
-2 f_{j}^{(n)} k_{j}^{(n-1)} \sin \left(\theta_{2 j-1}^{(n)}+\theta_{2 j}^{(n)}\right), \quad x_{n} \leq x \leq x_{j, \pi-\alpha_{j}^{(n)}}^{(n)}, \\
2 f_{j}^{(n)} k_{j}^{(n-1)} \sin \left(\theta_{2 j-1}^{(n)}+\theta_{2 j}^{(n)}\right), \quad x_{j, \pi-\alpha_{j}^{(n)}}^{(n)} \leq x \leq x_{n}+\left(f_{j}^{(n)}\right)^{-1} g_{n}, \\
-\min \left(\delta k_{j}^{(n)}, \frac{g_{n}}{2 x}\right) \frac{k_{j}^{(n-1)}}{4}\left(\sin 2 \theta_{2 j-1}^{(n)}+\sin 2 \theta_{2 j}^{(n)}\right), x>x_{n}+\left(f_{j}^{(n)}\right)^{-1} g_{n} .
\end{array}\right.
$$

It is assumed that $\delta \theta_{j}^{(n)}\left(x_{n}\right)<\pi / 2 ; x_{j, \pi-\alpha_{j}^{(n)}}^{(n)}$ is then defined as a minimal value of $x>x_{n}$ where $\delta \theta_{j}^{(n)}(x)$ reaches $\pi-\alpha_{j}^{(n)}$. We adopt this definition in order to 
avoid making too many assumptions on what happens for $x \leq x_{n}$, although in the construction process that appears in the following sections it is straightforward to check that $\delta \theta_{j}^{(n)}(x)$ does not reach $\pi-\alpha_{j}^{(n)}$ for $x \leq x_{n}$. Similarly, in the actual construction we will always have $x_{n}<<\left(f_{j}^{(n)}\right)^{-1} g_{n}$. As usual, $\delta k_{j}^{(n)}=\tilde{g}_{n}^{-1} f_{j}^{(n)}$. For simplicity, we are also going to assume that

$$
\left\|V^{(n)}\right\|_{L^{\infty}\left(\mathbb{R}^{+}\right)} \leq \frac{1}{2} \min _{j}\left\{\left(k_{j}^{(n)}\right)^{2}\right\} .
$$

We also suppose that the condition (2.8) of the Splitting Lemma holds:

$$
4 \sum_{j} f_{j}^{(n)} k_{j}^{(n-1)}<\frac{1}{3} a_{n}, \quad g_{n}>>1,2^{n} g_{n} a_{n}^{-2} \sum_{j=1}^{2^{n-1}} f_{j}^{(n)}<<1, \quad \delta k_{j}^{(n)}<\frac{1}{12} a_{n}
$$

for all $j$. The potential $V^{(n)}(x)$ on $\left(0, x_{n}\right)$ comes from the previous steps, but for now we make no more specific assumptions about it. Recall our notation $a_{n+1}=$ $\min _{j, j^{\prime}}\left|k_{j}^{(n)}-k_{j^{\prime}}^{(n)}\right|$. Let $\tilde{x}_{n}$ be such that for any $x \geq \tilde{x}_{n}$,

$$
\left|V^{(n)}(x)\right| \leq \frac{1}{8} a_{n+1} \min _{j}\left\{\left(k_{j}^{(n)}\right)\right\} .
$$

Also, take $\tilde{x}_{n}>g_{n} \max _{j}\left\{\left(f_{j}^{(n)}\right)^{-1}\right\}$ for convenience.

Lemma 3.1. Assume that $V^{(n)}(x)$ is given by (3.1) for $x>x_{n}$. Then

$$
\frac{\partial \theta}{\partial k}\left(x, k_{j}^{(n)}\right) \geq P_{n}\left(\frac{x}{\tilde{x}_{n}}\right)^{\beta\left(k_{j}^{(n)}\right)}
$$

for any $x \geq 2 \tilde{x}_{n}$. Here $\beta(k)=\frac{k_{j}^{(n-1)} g_{n}}{16 k}$. Moreover, if $k$ is such that

$$
\left|k-k_{j}^{(n)}\right| \leq \frac{1}{4} a_{n+1},
$$

then

$$
\frac{\partial \theta}{\partial k}(x, k) \leq D_{n}\left(1+\left(\frac{x}{\tilde{x}_{n}}\right)^{\beta(k)}\right)
$$

for any $x$. Here $D_{n}, P_{n}$ are positive constants defined only in terms of $V^{(n)}(x)$ for $x \leq x_{n}, x_{n},\left\{f_{j}^{(n)}\right\}, a_{n+1}$ and $g_{n}$.

Proof. It is clear that the value of $\tilde{x}_{n}$ depends only on $x_{n}, f_{j}^{(n)}, a_{n+1}$ and $g_{n}$ and can be easily estimated in terms of these quantities:

$$
\tilde{x}_{n} \leq C \max \left(2^{n} g_{n} a_{n+1}^{-1}, x_{n}+g_{n} \max _{j}\left\{\left(f_{j}^{(n)}\right)^{-1}\right\}\right) .
$$

Notice that from 2.3 it follows that

$$
\left(\frac{\partial \theta}{\partial k}(x, k)\right)^{\prime}=1+\frac{1}{k^{2}} V(x)(\sin \theta(x, k))^{2}-\frac{1}{k} V(x) \sin 2 \theta(x, k)\left(\frac{\partial \theta}{\partial k}(x, k)\right) .
$$

Along with the boundary condition $\frac{\partial \theta}{\partial k}(0, k)=0$ (which follows from the fact that we consider solutions satisfying fixed boundary conditions for all $k$ ) this implies in 
our setting

$$
\frac{\partial \theta}{\partial k}(x, k)=\int_{0}^{x} e^{-\frac{1}{k} \int_{y}^{x} V^{(n)}(s) \sin (2 \theta(s, k)) d s}\left(1+\frac{1}{k^{2}} V^{(n)}(y)(\sin \theta)^{2}\right) d y .
$$

For any $k$ satisfying the assumption (3.6) of the lemma we have for $\tilde{x}_{n} \leq y \leq x$ by an estimate parallel to that in Lemma 2.4 and using (3.4) and (3.3),

$$
\begin{aligned}
\int_{y}^{x} V^{(n)}(y) \sin 2 \theta(y, k) d y= & -\frac{k_{j}^{(n-1)} g_{n}}{16} \int_{y}^{x} \frac{\cos \left(2 \theta(s, k)-2 \theta_{j}^{(n)}(s)\right)}{s} d s \\
& +O\left(2^{n} a_{n+1}^{-2} g_{n} \sum_{j} f_{j}^{(n)}\right) .
\end{aligned}
$$

Equations (3.8), 3.9) together imply a very rough estimate

$$
\frac{\partial \theta}{\partial k}(x, k) \leq C \tilde{x}_{n} e^{\frac{1}{k} \int_{0}^{\tilde{x}_{n}}\left|V^{(n)}(y)\right| d y}+C e^{C 2^{n} a_{n+1}^{-2} g_{n} \sum_{j} f_{j}^{(n)}} \int_{\tilde{x}_{n}}^{x} e^{\beta(k) \log (x / y)} d y,
$$

where $C$ is a universal constant. Similarly,

$$
\frac{\partial \theta}{\partial k}\left(x, k_{j}^{(n)}\right) \geq C_{1} e^{-C 2^{n} a_{n+1}^{-2} g_{n} \sum_{j} f_{j}^{(n)}} \int_{\tilde{x}_{n}}^{x} e^{\beta\left(k_{j}^{(n)}\right) \log (x / y)} d y .
$$

Notice that

$$
\int_{\tilde{x}_{n}}^{x} e^{\beta(k) \log (x / y)} d y=\frac{\tilde{x}_{n}}{\beta(k)-1}\left(\left(\frac{x}{\tilde{x}_{n}}\right)^{\beta(k)}-\frac{x}{\tilde{x}_{n}}\right) .
$$

The parameter $\beta(k)$ is large because of $g_{n}$, and we can assume for simplicity $\beta(k)>$ 2 for all $k$ of interest (lying in a compact set where all $k_{j}^{(n)}$ lie). Then we get the result of the lemma. We can take, for example,

$$
\begin{gathered}
D_{n}=C \tilde{x}_{n}^{2} e^{\frac{1}{k} \int_{0}^{\tilde{x}_{n}}\left|V^{(n)}(y)\right| d y+C 2^{n} a_{n+1}^{-2} g_{n} \sum_{j} f_{j}^{(n)}}, \\
P_{n}=C_{1} \frac{\tilde{x}_{n}}{\beta\left(k_{j}^{(n)}\right)-1} e^{-C 2^{n} a_{n+1}^{-2} g_{n} \sum_{j} f_{j}^{(n)}} .
\end{gathered}
$$

Now we prove the principal result of this section.

Lemma 3.2 (Continuity Lemma). Choose $\tilde{g}_{n+1}$ so that $\tilde{g}_{n+1}^{1 / 4} \geq 2^{n} D_{n} P_{n}^{-1}$. Then there exists $x_{n}^{\prime}$ such that for any $x_{n+1}>x_{n}^{\prime}$ the following holds. If

$$
\left|k-k_{j}^{(n)}\right| \leq 2 \tilde{g}_{n+1}^{-1 / 2}\left(\frac{\partial \theta}{\partial k}\left(x_{n+1}, k_{j}^{(n)}\right)\right)^{-1},
$$

then

$$
R(x, k)^{2}=R\left(x, k_{j}^{(n)}\right)^{2}\left(1+O\left(\tilde{g}_{n+1}^{-1 / 4}\right)\right)
$$

and

$$
\frac{\partial \theta}{\partial k}(x, k)=\frac{\partial \theta}{\partial k}\left(x, k_{j}^{(n)}\right)\left(1+O\left(\tilde{g}_{n+1}^{-1 / 4}\right)\right)
$$

for all $x \leq x_{n+1}$. 
Proof. From (3.5) it follows that $\frac{\partial \theta}{\partial k}\left(x, k_{j}^{(n)}\right) \rightarrow \infty$ as $x \rightarrow \infty$, and thus we can choose $x_{n}^{\prime}$ so that for any $x_{n+1} \geq x_{n}^{\prime}$ the values of $k$ satisfying (3.12) also satisfy (3.6). Then Lemma 3.1 applies, and thus we have for any $x$,

$$
\frac{\frac{\partial \theta}{\partial k}(x, k)}{\frac{\partial \theta}{\partial k}\left(x_{n+1}, k_{j}^{(n)}\right)} \leq D_{n} P_{n}^{-1}\left(1+\left(\frac{x}{\tilde{x}_{n}}\right)^{\beta(k)}\right)\left(\frac{x_{n+1}}{\tilde{x}_{n}}\right)^{-\beta\left(k_{j}^{(n)}\right)} .
$$

Hence, by definition of $\tilde{g}_{n+1}$, we have

$$
\left|\theta(x, k)-\theta\left(x, k_{j}^{(n)}\right)\right| \leq 2^{-n} \tilde{g}_{n+1}^{-1 / 4}\left(1+\left(\frac{x}{\tilde{x}_{n}}\right)^{\beta(k)}\right)\left(\frac{x_{n+1}}{\tilde{x}_{n}}\right)^{-\beta\left(k_{j}^{(n)}\right)} .
$$

Recall from (2.2) that

$$
R(x, k)^{2} / R\left(x, k_{j}^{(n)}\right)^{2}=e^{\frac{1}{k} \int_{0}^{x} V^{(n)}(y) \sin 2 \theta(y, k) d y-\frac{1}{k_{j}^{(n)}} \int_{0}^{x} V^{(n)}(y) \sin 2 \theta\left(y, k_{j}^{(n)}\right) d y} .
$$

First of all, we can replace $1 / k$ with $1 / k_{j}^{(n)}$ in the first term in the exponent at the expense of creating an error factor of size at most

$$
e^{C\left|k-k_{j}^{(n)}\right|} \int_{0}^{x_{n+1}}\left|V^{(n)}(y)\right| d y
$$

for $x \leq x_{n+1}$. From (3.12) and a bound (3.5) of Lemma 3.1 it is clear that we can choose $x_{n}^{\prime}$ so that this factor does not exceed $1+O\left(\tilde{g}_{n+1}^{-1 / 4}\right)$. Next, we need to bound

$$
\frac{1}{k_{j}^{(n)}} \int_{0}^{x} V^{(n)}(y)\left|\theta(y, k)-\theta\left(y, k_{j}^{(n)}\right)\right| d y .
$$

Using (3.15), we can estimate the expression (3.16) for $x \leq x_{n+1}$ by

$$
C 2^{-n} \tilde{g}_{n+1}^{-1 / 4} x_{n+1}^{-\beta\left(k_{j}^{(n)}\right)} \tilde{x}_{n}^{\beta\left(k_{j}^{(n)}\right)-\beta(k)} \int_{0}^{x_{n+1}} y^{\beta(k)}\left|V^{(n)}(y)\right| d y .
$$

The contribution to (3.17) coming from the integral over $\left[0, \tilde{x}_{n}\right]$ can be made $O\left(\tilde{g}_{n+1}^{-1 / 4}\right)$ by the choice of $x_{n}^{\prime}$. Using an inequality $\left|V^{(n)}(y)\right| \leq C 2^{n} \beta(k) y^{-1}$ for $y>\tilde{x}_{n}$, we estimate the remaining part of (3.17) by $C \tilde{g}_{n+1}^{-1 / 4}\left(x_{n+1} / \tilde{x}_{n}\right)^{\beta(k)-\beta\left(k_{j}^{(n)}\right)}$. Notice that, from the definition of $\beta(k)$, we have

$$
\left|\beta(k)-\beta\left(k_{j}^{(n)}\right)\right| \leq C g_{n}\left|k-k_{j}^{(n)}\right| \leq C g_{n} \tilde{g}_{n+1}^{-1 / 2}\left(\frac{\partial \theta}{\partial k}\left(x_{n+1}, k_{j}^{(n)}\right)\right)^{-1} .
$$

By (3.5) of Lemma 3.1 and the definition of $\tilde{g}_{n+1}$, the right-hand side in (3.18) does not exceed $C\left(x_{n+1} / \tilde{x}_{n}\right)^{-\beta\left(k_{j}^{(n)}\right)}$. Thus,

$$
\left(\frac{x_{n+1}}{\tilde{x}_{n}}\right)^{\beta(k)-\beta\left(k_{j}^{(n)}\right)} \leq e^{C\left(\log \left(x_{n+1} / \tilde{x}_{n}\right)\right)\left(x_{n+1} / \tilde{x}_{n}\right)^{-\beta\left(k_{j}^{(n)}\right)}},
$$

which is bounded by some fixed universal constant. Combining the estimates, we see that if we choose $x_{n}^{\prime}$ to satisfy all of the above requirements, then (3.13) holds for any $x \leq x_{n+1}$, provided that $x_{n+1} \geq x_{n}^{\prime}$. 
Notice also that from (3.15) and (3.19), it follows that for $x \in\left(0, x_{n+1}\right)$ we have $\left|\theta(x, k)-\theta\left(x, k_{j}^{(n)}\right)\right| \leq C \tilde{g}_{n+1}^{-1 / 4}$. Thus (3.14) follows from (3.13) and (3.8), which can also be written as

$$
\frac{\partial \theta}{\partial k}(x, k)=R(x, k)^{-2} \int_{0}^{x} R(y, k)^{2}\left(1+\frac{1}{k^{2}} V^{(n)}(y)(\sin \theta)^{2}\right) d y .
$$

We used (3.2) in the last estimate.

Equation (3.20) provides a relation between the derivative of the Prüfer angle and the amplitude, which is going to be important for the construction. We remark that a similar formula appeared in a work by Carmona [1, but the angle and amplitude are defined slightly differently in [1] and the resulting relationship is not as convenient for our purpose.

\section{A BRICK OF CONSTRUCTION}

Our goal is to organize a Cantor-like process, splitting each eigenvalue we have at the $n$th step into two at the $(n+1)$ th step. To do this we need to see how norm splitting works in a more general setting than the Splitting Lemma, which starts at the origin. The needed estimates are provided by the following lemma, which helps to connect different steps.

Assume that for some $j$, we have for $k$ satisfying

$$
\left|k-k_{j}^{(n)}\right| \leq 2 \tilde{g}_{n+1}^{-1 / 2}\left(\frac{\partial \theta}{\partial k}\left(x_{n+1}, k_{j}^{(n)}\right)\right)^{-1}
$$

the bounds of the Continuity Lemma:

$$
R(x, k)^{2}=R\left(x, k_{j}^{(n)}\right)^{2}\left(1+O\left(\tilde{g}_{n+1}^{-1 / 4}\right)\right)
$$

and

$$
\frac{\partial \theta}{\partial k}(x, k)=\frac{\partial \theta}{\partial k}\left(x, k_{j}^{(n)}\right)\left(1+O\left(\tilde{g}_{n+1}^{-1 / 4}\right)\right)
$$

for all $x \leq x_{n+1}$. Set

$$
\begin{aligned}
f_{j}^{(n+1)} & =\tilde{g}_{n+1}^{1 / 2}\left(\frac{\partial \theta}{\partial k}\left(x_{n+1}, k_{j}^{(n)}\right)\right)^{-1}, \\
\delta k_{j}^{(n+1)} & =\tilde{g}_{n+1}^{-1 / 2}\left(\frac{\partial \theta}{\partial k}\left(x_{n+1}, k_{j}^{(n)}\right)\right)^{-1},
\end{aligned}
$$

and let $k_{2 j}^{(n+1)}, k_{2 j-1}^{(n+1)}$ be the ends of the interval of size $\delta k_{j}^{(n+1)}$ centered at $k_{j}^{(n)}$. For $x>x_{n+1}$, define the potential $V^{(n+1)}(x)$ to be given by (3.1) with $n+1$ replacing $n$. We are going to assume also that the conditions (3.3) of the Splitting Lemma are satisfied (with $n$ replaced by $n+1$ in our current situation). Define a parameter $A_{j}^{(n)}$ by

$$
A_{j}^{(n)}=\frac{\partial \theta}{\partial k}\left(x_{n+1}, k_{j}^{(n)}\right) R\left(x_{n+1}, k_{j}^{(n)}\right)^{2} .
$$


We have

Lemma 4.1 (Connection Lemma). Under the above assumptions, $k_{2 j-1}^{(n+1)}$ and $k_{2 j}^{(n+1)}$ are eigenvalues of $H_{V^{(n+1)}}$, and the norms of the corresponding eigenfunctions on $\left(x_{n+1}, \infty\right)$ satisfy

$$
\left\|R\left(x, k_{2 j, 2 j-1}^{(n+1)}\right)\right\|_{L^{2}\left(x_{n+1}, \infty\right)}^{2}=A_{j}^{(n)}\left(1+O\left(\tilde{\mathcal{E}}_{n+1}, \tilde{g}_{n+1}^{-1 / 4}\right)\right) .
$$

Proof. To simplify notation, let us assume without loss of generality that $j=1$. According to our assumptions, $\delta \theta_{1}^{(n+1)}\left(x_{n+1}\right)=\tilde{g}_{n+1}^{-1 / 2}\left(1+O\left(\tilde{g}_{n+1}^{-1 / 4}\right)\right)$. If $\delta \theta_{1}^{(n+1)}\left(x_{n+1}\right)$ were zero, we would be exactly in a situation of the Splitting Lemma construction and would have two eigenfunctions of norm $\sim 2\left(f_{j}^{(n+1)}\right)^{-1} R\left(x_{n+1}, k_{j}^{(n)}\right)^{2}$. We have to take into account the fact that on the interval where $\delta \theta_{1}^{(n+1)}(x) \in$ $\left[0, \delta \theta_{1}^{(n+1)}\left(x_{n+1}\right)\right]$ a significant decay takes place in the Splitting Lemma, and we lose this decay in our situation.

Consider an auxiliary problem for $\bar{R}, \delta \bar{\theta}$ satisfying the same equations (2.10), (2.11) as $R\left(x, k_{1}^{(n+1)}\right), \delta \theta_{1}^{(n+1)}$ but with $\bar{V}^{(n+1)}$ given by (2.7) with $n+1$ instead of $n$. In other words, consider the case of the $(n+1)$ st step potential starting right away at the origin. The Small Angle and Splitting Lemmas imply that

$$
\left\|\bar{R}\left(x, k_{1}^{(n+1)}\right)\right\|_{L^{2}\left(x_{\delta \theta_{1}\left(x_{n+1}\right)}, \infty\right)}^{2}=\left(f_{1}^{(n+1)}\right)^{-1}\left(1+O\left(\tilde{\mathcal{E}}_{n+1}, \tilde{g}_{n+1}^{-1 / 2} g_{n+1}\right)\right) .
$$

But the same estimates as in these lemmas apply to $R\left(x, k_{1}^{(n+1)}\right), \delta \theta_{1}$ on $\left(x_{n+1}, \infty\right)$, by identical arguments. The only adjustment we need to make is by a factor

$$
R\left(x_{n+1}, k_{1}^{(n+1)}\right)^{2} / \bar{R}\left(x_{\delta \theta_{1}\left(x_{n+1}\right)}, k_{1}^{(n+1)}\right)^{2} .
$$

By Lemma 2.6 (Small Angle Lemma),

$$
\begin{aligned}
& \bar{R}\left(x_{\delta \theta_{1}\left(x_{n+1}\right)}, k_{1}^{(n+1)}\right)^{2} \\
& \quad=\left(\frac{\delta k_{1}^{(n+1)}}{\delta \theta_{1}^{(n+1)}\left(x_{n+1}\right) f_{1}^{(n+1)}+\delta k_{1}^{(n+1)}}\right)\left(1+O\left(\mathcal{E}_{n+1}, \tilde{g}_{n+1}^{-1} g_{n+1}\right)\right) .
\end{aligned}
$$

According to (4.7), (4.8) we have

$$
\begin{aligned}
& \left\|R\left(x, k_{1,2}^{(n+1)}\right)\right\|_{L^{2}\left(x_{n+1}, \infty\right)}^{2} \\
& \quad=\frac{R\left(x_{n+1}, k_{1}^{(n)}\right)^{2}}{f_{1}^{(n+1)}}\left(\frac{\delta \theta_{1}^{(n+1)}\left(x_{n+1}\right) f_{1}^{(n+1)}}{\delta k_{1}^{(n+1)}}+1\right)\left(1+O\left(\tilde{\mathcal{E}}_{n+1}, \tilde{g}_{n+1}^{-1 / 4}\right)\right) .
\end{aligned}
$$

We utilized assumption (4.1) in the error estimate, and also used $\tilde{g}_{n+1}^{-1} g_{n+1}<$ $C \tilde{g}_{n+1}^{-1 / 4}$. Because of the assumption (4.2), we have

$$
\frac{\delta \theta_{1}^{(n+1)}\left(x_{n+1}\right)}{\delta k_{1}^{(n+1)}}=\frac{\partial \theta}{\partial k}\left(x_{n+1}, k_{1}^{(n)}\right)\left(1+O\left(\tilde{g}_{n+1}^{-1 / 4}\right)\right) .
$$

The term $\left(f_{1}^{(n+1)}\right)^{-1} R\left(x_{n+1}, k_{1}^{(n)}\right)^{2}=A_{j}^{(n)} \tilde{g}_{n+1}^{-1 / 2}$ and hence contributes a correction of higher order. Thus by definition of $A_{j}^{(n)}$ and (4.10), (4.9) leads exactly to (4.6). 
The usefulness of Lemma 4.1 becomes clear if one looks at (3.20), which in particular implies

$$
\frac{\partial \theta}{\partial k}(x, k)=R(x, k)^{-2}\|R(y, k)\|_{L^{2}(0, x)}^{2}\left(1+O\left(\|V\|_{L^{\infty}}\right)\right) .
$$

Thus in the asymptotic regime where $V$ is small, one can expect, informally,

$$
\frac{\partial \theta}{\partial k}(x, k) \sim R(x, k)^{-2}\|R(y, k)\|_{L^{2}(0, x)}^{2},
$$

and the role of $A$ is played by $\|R(x, k)\|_{L^{2}(0, x)}^{2}$. Lemma 4.1 allows us to get two eigenvalues with the norm on $(x, \infty)$ the same as $A=\|R(x, k)\|_{L^{2}(0, x)}^{2}$. This will provide the mechanism for norm doubling in the asymptotic regime, which is crucial for the construction.

\section{The CONSTRUCTION}

Now we are ready to provide a precise description of the construction of our potential $V(x)$. In the beginning, we take a potential given by (2.7), such as in the Splitting Lemma with $n=1$. Start by choosing $\delta k_{1}^{(1)}, k_{1}^{(0)}, g_{1}$ and $f_{1}^{(1)}$. The precise choice of the parameters at the first step of the construction is not so important, but we are going to keep it similar to the subsequent steps. To satisfy (2.6) we choose $k_{1}^{(0)}$ sufficiently large and $\delta k_{1}^{(1)}$ small compared to $k_{1}^{(0)}$. We take $g_{1}$ large, impose a relationship $\delta k_{1}^{(1)}=\tilde{g}_{1}^{-1} f_{1}^{(1)}$ and take $e^{g_{1}} f_{1}^{(1)}$ small. The smallness of the latter number and $\delta k_{1}^{(1)}$ can be achieved by taking $f_{1}^{(1)}$ sufficiently small while keeping $g_{1}$ fixed. In particular, we take $g_{1}$ large enough and $f_{1}^{(1)}$ small enough for the conditions (3.3) of the Splitting to be satisfied. The only additional restriction we need to impose on the parameters comes from the condition $|V(x)| \leq h(x) /(1+x)$. By the monotonicity of $h$ and the definition of potential, the inequality

$$
2 f_{1}^{(1)} k_{1}^{(0)} \leq \frac{h\left(\left(f_{1}^{(1)}\right)^{-1} g_{1}\right)}{1+\left(f_{1}^{(1)}\right)^{-1} g_{1}}
$$

implies the needed bound for $x \geq\left(f_{1}^{(1)}\right)^{-1} g_{1}$. It can be rewritten as $h\left(\left(f_{1}^{(1)}\right)^{-1} g_{1}\right) \geq$ $2\left(g_{1}+f_{1}^{(1)}\right) k_{1}^{(0)}$, which holds for sufficiently small $f_{1}^{(1)}$. To simplify consideration for smaller $x$, we are going to assume without loss of generality that $h(x) /(1+x)$ is decreasing. Then (5.1) also implies the bound for $x<\left(f_{1}^{(1)}\right)^{-1} g_{1}$. The Splitting Lemma then gives us two eigenvalues at $k_{1,2}^{(1)}$ with corresponding eigenfunctions having norms $\left(f_{1}^{(1)}\right)^{-1}\left(1+O\left(e^{g_{1}} f_{1}^{(1)}, \tilde{g}_{1}^{-1 / 2} g_{1}\right)\right)$.

Let us now describe the induction process. We are going to assume that at the $n$th step we have the potential $V^{(n)}(x)$, in particular given by (3.1) on $\left(x_{n}, \infty\right)$. We assume that this potential leads to the eigenvalues $k_{j}^{(n)}, j=1, \ldots, 2^{n}$ and the following holds:

1. The norms of the corresponding eigenfunctions satisfy for every $j$,

$$
\left\|R\left(x, k_{j}^{(n)}\right)\right\|_{L^{2}(0, \infty)}^{2} \leq C_{1} 2^{n} \prod_{l=1}^{n}\left(1+E r_{l}\right),
$$




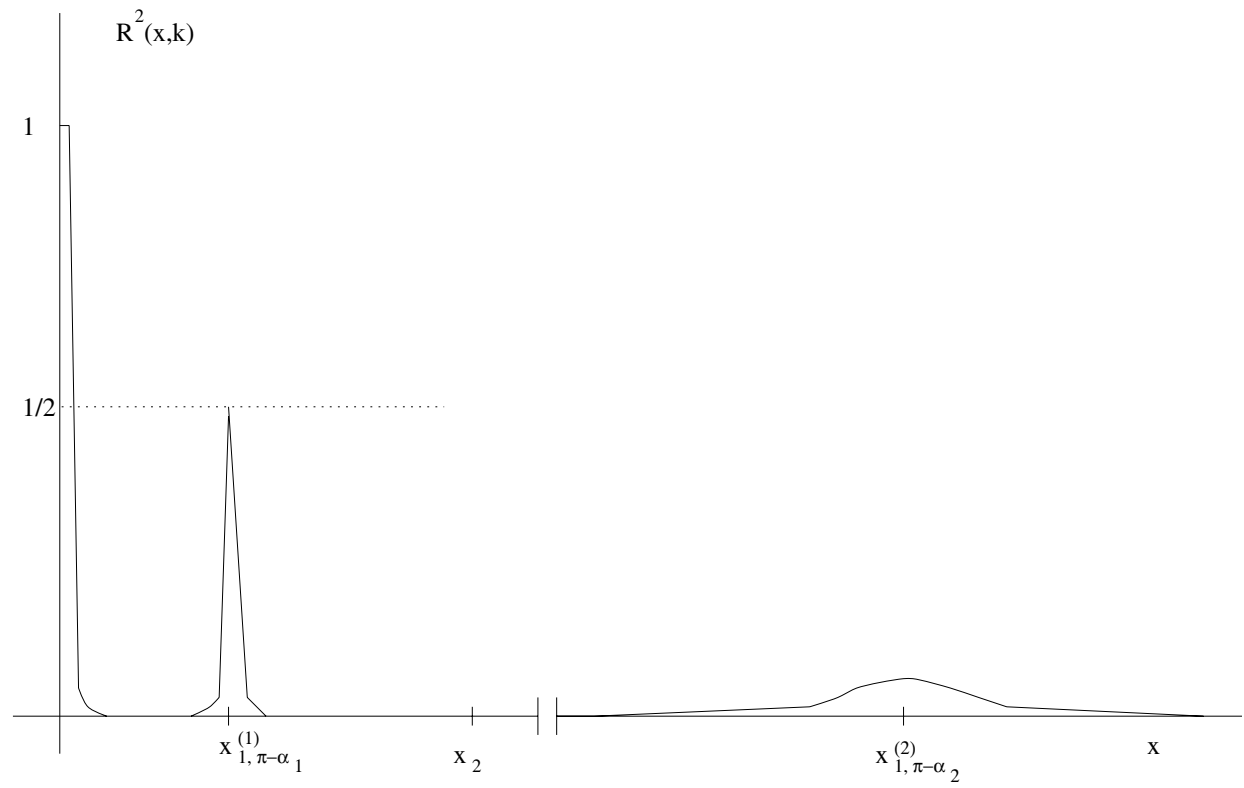

Figure 2. The structure of the eigenfunction at $k=k_{1}^{(n)}$. Only the first two stages are shown. The factor by which the eigenfunction grows from the splitting point $x_{n}$ to its top value at $x_{1, \pi-\alpha_{1}^{(n)}}^{(n)}$ is $\sim(1 / 2) \tilde{g}_{n}^{1 / 2}$. This can be seen from the argument in the Connection Lemma. Each bump contains roughly as much norm as everything before it.

where $\left|E r_{l}\right| \leq C_{2} 2^{-l}$, and $C_{1,2}$ are fixed constants which do not depend on $n, l$.

2. The angle derivative in energy satisfies for every $j$,

$$
\frac{\partial \theta}{\partial k}\left(x, k_{j}^{(n)}\right) \leq C_{1} 2^{n} R\left(x, k_{j}^{(n)}\right)^{-2} \prod_{l=1}^{n}\left(1+E r_{l}\right)
$$

for any $x$.

3. The conditions (3.3) of the Splitting Lemma are satisfied, and $\left|V^{(n)}(x)\right| \leq$ $h(x) /(1+x)$.

4. We assume that (2.6) holds; this will be nearly automatic because of the rapid decrease of $\delta k_{j}^{(n)}$.

Clearly for $n=1$ these assumptions are satisfied with $x_{n}=0$ and sufficiently large $C_{1,2} . C_{1}$, in particular, should be taken $\sim\left(f_{1}^{(1)}\right)^{-1}$ or larger. The fact that (5.3) is satisfied for $n=1$ follows from (5.2) and (3.20). The structure of the eigenfunctions that we are constructing is illustrated by Figure 2

Now let us make an induction step. Choose $\tilde{g}_{n+1}$ so that $\tilde{g}_{n+1}^{1 / 4}>2^{n} D_{n} P_{n}^{-1}$, where $D_{n}, P_{n}$ are given by (3.10), (3.11). We should also make sure that $g_{n+1}$ is large enough so that the second condition of (3.3) needed for the Splitting and Connection Lemmas holds; but this is basically automatic after the first step, since $g_{n}$ is virtually forced by construction to be an increasing sequence. We require 
in addition that $\tilde{g}_{n+1}^{-1 / 4} \leq C 2^{-n-1}$ (which is again basically automatic). For each $j=1, \ldots, 2^{n}$, choose

$$
f_{j}^{(n+1)}=\tilde{g}_{n+1}^{1 / 2}\left(\frac{\partial \theta}{\partial k}\left(x_{n+1}, k_{j}^{(n)}\right)\right)^{-1}, \quad \delta k_{j}^{(n+1)}=\tilde{g}_{n+1}^{-1 / 2}\left(\frac{\partial \theta}{\partial k}\left(x_{n+1}, k_{j}^{(n)}\right)\right)^{-1}
$$

where the splitting point $x_{n+1}$ remains the only parameter of the $(n+1)$ st step to be chosen. Define

$$
\tilde{f}_{n+1}=C a_{n+1}^{2} 2^{-3(n+1)} e^{-g_{n+1}} .
$$

One condition that we impose on $x_{n+1}$ is that $f_{j}^{(n+1)}$ defined by (5.4) are smaller than $\tilde{f}_{n+1}$ for every $j$. This can always be achieved by taking $x_{n+1}$ large enough because of 3.5 . Notice that such a choice ensures that $\tilde{\mathcal{E}}_{n+1} \leq C 2^{-n-1}$. Also, the constant $C$ in (5.5) is chosen so that the first, third and fourth conditions of (3.3) needed for the Splitting and Connection Lemmas at the $(n+1)$ st step hold.

The second condition imposed on $x_{n+1}$ is that Lemma 3.2 (Continuity Lemma) applies, that is, $x_{n+1}>x_{n}^{\prime}, x_{n}^{\prime}$ is defined in Lemma 3.2, and $\delta k_{j}^{(n)} \leq \frac{1}{12} a_{n+1}$ for all $j$.

The third condition comes from the constraint $\left|V^{(n+1)}(x)\right| \leq h(x) /(1+x)$. The potential $V^{(n+1)}$ on $\left[x_{n+1}, \infty\right)$ is going to be defined by (3.1) with $n+1$ instead of $n$, so the latter requirement is going to be satisfied if for every $j$,

$$
f_{j}^{(n+1)} \leq 2^{-n-1} \frac{h\left(x_{n+1}+\left(f_{j}^{(n+1)}\right)^{-1} g_{n+1}\right)}{x_{n+1}+\left(f_{j}^{(n+1)}\right)^{-1} g_{n+1}} .
$$

By (3.5) we have

$$
\left(\frac{\partial \theta}{\partial k}\left(x, k_{j}^{(n)}\right) / x\right) \stackrel{x \rightarrow \infty}{\longrightarrow} \infty
$$

(since we assume that $g_{n}$ is large enough). Thus, for large enough $x_{n+1}$ we have $x_{n+1} \leq\left(f_{j}^{(n+1)}\right)^{-1} g_{n+1}$ (recall that $f_{j}^{(n+1)}$ is defined by (5.4) $)$. Then the condition (5.6) reduces to

$$
2^{n+2} g_{n+1} \leq h\left(x_{n+1}+\left(f_{j}^{(n+1)}\right)^{-1} g_{n+1}\right) .
$$

This can always be achieved by taking $x_{n+1}$ sufficiently large while $g_{n+1}$ is fixed.

By the choice of $\delta k_{j}^{(n+1)}, x_{n+1}$ and $\tilde{g}_{n+1}$, the result of Lemma 3.2 (Continuity Lemma) applies to $k_{j}^{(n+1)}$, and therefore we can use Lemma 4.1 (Connection Lemma). Together, the Continuity and Connection Lemmas show that $k_{j}^{(n+1)}$ are eigenvalues with eigenfunctions satisfying

$$
\left\|R\left(x, k_{j}^{(n+1)}\right)\right\|_{L^{2}}^{2} \leq C_{1} 2^{n} \prod_{l=1}^{n}\left(1+E r_{l}\right)\left[\left(1+C \tilde{g}_{n+1}^{-1 / 4}\right)+\left(1+C\left(\tilde{g}_{n+1}^{-1 / 4}+\tilde{\mathcal{E}}_{n+1}\right)\right)\right] .
$$

Here the first summand in the brackets comes from $\left(0, x_{n+1}\right)$ and was estimated by the induction assumption (5.2) and Continuity Lemma, while the second summand comes from $\left(x_{n+1}, \infty\right)$ and was estimated using (5.3), Continuity and Connection Lemmas. Altogether, we obtain

$$
\left\|R\left(x, k_{j}^{(n+1)}\right)\right\|_{L^{2}}^{2} \leq C_{1} 2^{n+1} \prod_{l=1}^{n+1}\left(1+E r_{l}\right),
$$


where $E r_{n+1} \leq C_{2} 2^{-n-1}$ and thus satisfies the required bound. Next,

$$
\begin{aligned}
\frac{\partial \theta}{\partial k}\left(x, k_{j}^{(n+1)}\right)= & \frac{\partial \theta}{\partial k}\left(x_{n+1}, k_{j}^{(n+1)}\right) \frac{R\left(x_{n+1}, k_{j}^{(n+1)}\right)^{2}}{R\left(x, k_{j}^{(n+1)}\right)^{2}} \\
& +\int_{x_{n+1}}^{x} \frac{R\left(y, k_{j}^{(n+1)}\right)^{2}}{R\left(x, k_{j}^{(n+1)}\right)^{2}}(1+O(|V(y)|)) d y .
\end{aligned}
$$

By the induction assumption and Continuity Lemma, the first summand does not exceed

$$
R\left(x, k_{j}^{(n+1)}\right)^{-2} C_{1} 2^{n} \prod_{l=1}^{n}\left(1+E r_{l}\right)\left(1+C \tilde{g}_{n+1}^{-1 / 4}\right) .
$$

The second summand, by the Connection Lemma, does not exceed

$$
R\left(x, k_{j}^{(n+1)}\right)^{-2} C_{1} 2^{n} \prod_{l=1}^{n}\left(1+E r_{l}\right)\left(1+C\left(\tilde{\mathcal{E}}_{n+1}+\tilde{g}_{n+1}^{-1 / 4}\right)\right)\left(1+C 2^{n} \tilde{f}_{n+1}\right) .
$$

Therefore, taking into account the above estimates on $\tilde{\mathcal{E}}_{n+1}, \tilde{g}_{n+1}^{-1 / 4}$ and the choice of $\tilde{f}_{n+1}$, we get that

$$
\frac{\partial \theta}{\partial k}\left(x, k_{j}^{(n+1)}\right)=R\left(x, k_{j}^{(n+1)}\right)^{-2} C_{1} 2^{n+1} \prod_{l=1}^{n+1}\left(1+E r_{l}\right),
$$

with $E_{n+1} \leq C_{2} 2^{-n-1}$. The third component of the induction step was already handled by the choice of $x_{n+1}$. The fourth component is easily satisfied, since by inspection of the argument it is clear that $\delta k_{j}^{(n)}$ decay rapidly in $n$. In fact we can assume freely that $g_{n}>2^{n}$ (there is no restriction on how large a $g_{n}$ we can choose at each step, only a bound from below). Since $f_{j}^{(n)} \leq C 2^{-3 n} e^{-g_{n}}$ for any $j$ and $\delta k_{j}^{(n)}$ is even smaller, we can clearly arrange for (2.6) to be valid. This concludes the induction step.

Notice that by construction, $\max _{j} \delta k_{j}^{(n+1)} \leq \tilde{f}_{n+1} \tilde{g}_{n+1}^{-1 / 2}$, and by definition of $a_{n+1}, \tilde{f}_{n+1} \leq 2^{-3 n} e^{-g_{n}}\left(\min _{j} \delta k_{j}^{(n)}\right)^{2}$. It is easy to see from these relations that for all sufficiently large $n$,

$$
\sum_{m=n+1}^{\infty} \max _{j}\left(\delta k_{j}^{(m)}\right) \leq \frac{1}{3} \min _{j}\left(\delta k_{j}^{(n)}\right) .
$$

Denote by $I_{j}^{(n)}$ the intervals with centers at $k_{j}^{(n)}$ of size $\frac{1}{3} \delta k_{j}^{(n)}$. Then by (5.8), for sufficiently large $n, 2 I_{j}^{(n)}$ are disjoint for different $j$ (where by $2 I_{j}^{(n)}$ we mean the intervals centered at $k_{j}^{(n)}$ and twice the size of $\left.I_{j}^{(n)}\right)$. At the same time, all eigenvalues $k_{l}^{(m)}$ that are generated from $k_{j}^{(n)}$ at a later step and their intervals $I_{l}^{(m)}$ lie in $I_{j}^{(n)}$ (these are the eigenvalues $k_{l}^{(m)}, l=(j-1) 2^{m-n}+1, \ldots, j 2^{m-n}$ for any $m>n)$. For the size of $I_{j}^{(n)}$ we have an estimate $\left|I_{j}^{(n)}\right| \leq C e^{-2^{n}}$ provided that we choose $g_{n}>2^{n}$ (the estimate is even stronger, but this will suffice).

Let us summarize our findings.

Theorem 5.1. Applying the construction described above we obtain a sequence of potentials $V^{(n)}(x)$ with the following properties. Each $V^{(n)}(x)$ satisfies $\left|V^{(n)}(x)\right| \leq$ 
$h(x) /(1+x)$. If $m>n$, then $V^{(m)}(x)=V^{(n)}(x)$ for $x \leq x_{n}$, where $\left\{x_{n}\right\}$ is a strictly increasing sequence tending to infinity as $n$ grows. For each $n$, the operator $H_{V^{(n)}}$ has $2^{n}$ eigenvalues $k_{j}^{(n)}, j=1, \ldots, 2^{n}$ such that for any $j$,

$$
\int_{0}^{\infty} R\left(x, k_{j}^{(n)}\right)^{2} d x \leq B^{-1} 2^{n},
$$

where $B>0$ is a universal constant independent of $n$. Moreover, for each $k_{j}^{(n)}$ we can define an interval $I_{j}^{(n)}$ centered at $k_{j}^{(n)}$ such that $\left|I_{j}^{(n)}\right| \leq C e^{-2^{n}}$ and for any $m>n$, all $k_{l}^{(m)}$ with $l=(j-1) 2^{m-n}+1, \ldots, j 2^{m-n}$ belong to $I_{j}^{(n)}$. At the same time, intervals $2 I_{j}^{(n)}, j=1, \ldots, 2^{n}$ for fixed $n$ are disjoint from each other.

Proof. We have already shown every aspect of the theorem except we have not directly stated (5.9). However this estimate follows directly from the first induction assumption, (5.2), since $E r_{l} \leq C_{2} 2^{-l}$.

\section{Singular continuous Spectrum}

In this section we prove Theorem 1.1. We are going to define the potential $V(x)$ by a condition $V(x)=V^{(n)}(x)$ for $x \leq x_{n}$. By Theorem 5.1 this defines unambiguously $V(x)$ on all positive semi-axes. We prove the following result, which implies Theorem 1.1.

Theorem 6.1. The singular continuous spectrum of the operator $H_{V}$ is nonempty.

Proof. Let us denote the spectral measure of $H_{V}$ by $\mu$, and spectral measures of $H_{V^{(n)}}$ by $\mu_{n}$. Notice that since $V=V^{(n)}$ for $x<x_{n}$ and $x_{n} \rightarrow \infty$, the operator $H_{V^{(n)}}$ converges to $H_{V}$ in a strong resolvent sense. The strong resolvent convergence implies weak convergence of $\mu_{n}$ to $\mu$ (see, e.g., 19], Theorem VII.20). Consider any interval $I_{j}^{(n)}$ with $n$ sufficiently large. Then by Theorem 5.1 and Lemma [2.1, we have $\mu_{m}\left(I_{j}^{(n)}\right) \geq B 2^{-n}$ for each $m>n$. This implies by weak convergence that $\mu\left(2 I_{j}^{(n)}\right) \geq B 2^{-n}$ for every $n$ and $j=1, \ldots, 2^{n}$. Assume that $\mu$ does not have a singular continuous component, so that $\mu=\mu^{\mathrm{ac}}+\mu^{\mathrm{pp}}$. Take a small number $\epsilon<B / 10$. Then there exists a $\delta_{\epsilon}$ such that if $|S|<\delta_{\epsilon}, \mu^{\text {ac }}(S)<\epsilon$ (we denote here by $|S|$ the Lebesgue measure of the set $S$ ). Choose $n_{1}$ so that for any $n>n_{1}$,

$$
\sum_{j=1}^{2^{n}}\left|2 I_{j}^{(n)}\right| \leq C 2^{n+1} e^{-2^{n}}<\delta_{\epsilon} .
$$

Then

$$
\mu^{\mathrm{ac}}\left(\bigcup_{j=1}^{2^{n}}\left(2 I_{j}^{(n)}\right)\right) \leq \epsilon<B / 10
$$

for any $n>n_{1}$. Let $\mu^{\mathrm{pp}}=\sum_{l=1}^{\infty} \rho_{l} \delta\left(x-x_{l}\right)$. Pick $N_{\epsilon}$ so that $\sum_{l=N_{\epsilon}}^{\infty} \rho_{l}<\epsilon$. Choose $n_{2}$ so that $2^{n_{2}}>N_{\epsilon}$. Then for any $n>n_{2}$, there are at least $2^{n}-2^{n_{2}}$ intervals $2 I_{j}^{(n)}$ which do not contain any of the $x_{l}$ with $l \leq N_{\epsilon}$. Denote the set of such intervals by $Q_{n}$. Then we have

$$
\mu^{\mathrm{pp}}\left(\bigcup_{j \in Q_{n}}\left(2 I_{j}^{(n)}\right)\right) \leq \epsilon<B / 10
$$


for any $n>n_{2}$. At the same time, by Theorem 5.1, we have

$$
\mu\left(\bigcup_{j \in Q_{n}}\left(2 I_{j}^{(n)}\right)\right) \geq B / 2
$$

if $n>n_{2}$. For $n \geq \max \left(n_{1}, n_{2}\right)$, this gives us a contradiction with (6.1), (6.2) and our assumption on the absence of a singular continuous component.

Theorem 1.1 follows from Theorem 6.1 immediately since from Theorem 5.1 we know that $|V(x)| \leq h(x) /(1+x)$.

\section{Asymptotic inCOMPlETENESS OF WAVE OPERATORS}

In [5, it was proved that the Möller wave operators exist if $V \in L^{p}, p<2$, and in addition $\lim _{x \rightarrow \infty} \int_{0}^{x} V(s) d s$ exists. Therefore, to prove Theorem 1.2, we only need to show that the potential $V(x)$, constructed in the previous sections, can be chosen to be conditionally integrable.

Proof of Theorem 1.2. Consider one of the components of $V^{(n)}(x)$, say $V_{j}^{(n)}(x)$, on $\left(x_{n}, \infty\right)$. Simple integration by parts similar to Lemma 2.4 shows that for any $x$,

$$
\left|\int_{x_{n}}^{x} V_{j}^{(n)}(y) d y\right| \leq C\left(f_{j}^{(n)}+\int_{x_{n}}^{\infty} \tilde{f}_{j}^{(n)}(y)\left|V^{(n)}(y)\right| d y\right),
$$

where similarly to what we had before in $(\underline{2.34}), \tilde{f}_{j}^{(n)}$ is given by

$$
\tilde{f}_{j}^{(n)}(y)= \begin{cases}-f_{j}^{(n)}, & x_{n} \leq x \leq x_{j, \pi-\alpha_{j}^{(n)}}^{(n)}, \\ f_{j}^{(n)}, & x_{j, \pi-\alpha_{j}^{(n)}}^{(n)} \leq x \leq x_{n}+\left(f_{j}^{(n)}\right)^{-1} g_{n}, \\ -\min \left(\delta k_{j}^{(n)}, \frac{g_{n}}{2 x}\right) \frac{k_{j}^{(n-1)}}{4}, & x>x_{n}+\left(f_{j}^{(n)}\right)^{-1} g_{n} .\end{cases}
$$

Since we also have $\left|V^{(n)}(y)\right| \leq C \sum_{j} \tilde{f}_{j}^{(n)}(y)$, a simple computation gives that

$$
\left|\int_{x_{n}}^{x} V^{(n)}(y) d y\right| \leq C 2^{n} g_{n} \sum_{j} f_{j}^{(n)}
$$

for any $x>x_{n}$. According to our choice of parameters in the construction, in particular $\tilde{f}_{n}$, the bound on the right-hand side does not exceed $C 2^{-n}$. Summing up the contributions from $\left(x_{n}, x_{n+1}\right)$ for different $n$, we get the result.

\section{Absence of singular continuous spectrum FOR THE COULOMB RATE OF DECAY}

Our goal in this section is to prove Theorem 1.3. We will need two useful general facts. First, denote by $\mu^{(L)}$ the spectral measure corresponding to the operator with potential $V(x) \chi_{[0, L]}(x)$. Then

$$
\frac{d \mu^{(L)}}{d x}(k)=\frac{2 k^{2}}{\pi R^{2}(L, k)} .
$$

We refer to [18] for this relation. Secondly, assume that $V_{1}$ and $V_{2}$ are bounded, $\left|V_{1}(x)\right|,\left|V_{2}(x)\right| \leq B$, and $V_{1}(x)=V_{2}(x)$ for $0 \leq x \leq L$. Denote by $\mu_{1,2}$ the spectral 
measures of $H_{V_{1,2}}$ respectively. Fix some compact interval $I$ and arbitrary $M, \sigma>0$. Then for any $\epsilon>L^{-\frac{1}{1+\sigma}}$, we have

$$
\mu_{1}(E-\epsilon, E+\epsilon) \geq \mu_{2}\left(E-\frac{\epsilon}{2}, E+\frac{\epsilon}{2}\right)-C(I, \sigma, B, M) \epsilon^{M} .
$$

We refer to Appendix 2 of 10 for the proof.

Next, we prove

Lemma 8.1. Assume that the function $G(x)$ satisfies $\left|G^{\prime}(x)\right| \leq \frac{B}{1+x}$. Then

$$
\left|\int_{0}^{L} \frac{\sin (\delta k x+G(x))}{1+x} d x\right| \leq C_{1} \log (\delta k)^{-1}+C_{2} .
$$

The constants $C_{1,2}$ depend only on the constant $B$ in the bound for the function $G(x)$.

Proof. It is sufficient to estimate $\int_{0}^{L} \exp (i \delta k x+G(x))(1+x)^{-1} d x$. Introduce $H(x)=$ $\int_{x}^{\infty} \exp (i \delta k y)(1+y)^{-1} d y$. Note that

$$
|H(x)| \leq 2 \int_{x}^{x+\pi(\delta k)^{-1}} y^{-1} d y=2 \log \left(1+\frac{\pi(\delta k)^{-1}}{x}\right) .
$$

Now,

$$
\left|\int_{0}^{\pi(\delta k)^{-1}} \frac{\exp (i \delta k+i G(x))}{1+x} d x\right| \leq \log \left(\pi(\delta k)^{-1}\right) .
$$

On the other hand,

$$
\begin{aligned}
& \left|\int_{\pi(\delta k)^{-1}}^{L} \frac{\exp (i \delta k+i G(x))}{1+x} d x\right| \\
& \quad=|H(x) \exp (i G(x))|_{\pi(\delta k)^{-1}-}^{L} \int_{\pi(\delta k)^{-1}}^{L} i H(x) G^{\prime}(x) \exp (i G(x)) d x \mid \\
& \leq 4 \log 2+2 B \int_{\pi(\delta k)^{-1}}^{L} \frac{\pi(\delta k)^{-1}}{x^{2}} d x \leq 4 \log 2+2 B .
\end{aligned}
$$

Combining the estimates we get the required result.

Let us now proceed to the first key observation: a certain forbidden scenario in terms of the growth (or decay) of the Prüfer amplitude at a set of energies. For the rest of this section, we fix some $\beta<1, M>1$ and $\sigma>0$. Given an energy scale $\epsilon$, we will always relate to it the length scale $L=\epsilon^{-1-\sigma}$.

We will consider energies in a fixed compact interval $I=[a, b], a>0$. Choose a sufficiently small $\epsilon>0$ (it will be clear from the estimates what is meant by this; how small will only depend on the constant $B$ in the bound on $V, I, \beta$ and $\sigma$ ). 
Consider $N$ resonant energies $k_{j} \in I$ such that the following two conditions are satisfied. First, for each $k_{j}$ we have

$$
\left|\int_{0}^{L} V(x) \sin 2 \theta\left(x, k_{j}\right) d x\right| \geq \frac{a(1-\beta)}{100} \log \epsilon^{-1},
$$

where as usual $L=\epsilon^{-1-\sigma}$. Second, we demand that

$$
\min _{j_{1}, j_{2}}\left|k_{j_{1}}-k_{j_{2}}\right| \geq \epsilon^{1 / N^{2}},
$$

so that the resonant energies are sufficiently separated. We are going to show that in this case there is an upper bound on how large $N$ can be, which depends only on $B, I, \beta$ and $\sigma$.

Proposition 8.2. Assume that the potential $V(x)$ satisfies $|V(x)| \leq B(1+x)^{-1}$. There exists $\tilde{\epsilon}(B, I, \sigma, \beta)>0$ such that for any $0<\epsilon<\tilde{\epsilon}$, there can be no more than $\tilde{N}(B, I, \sigma, \beta)$ energies in I for which (8.4) and (8.5) hold.

Proof. Let us introduce

$$
e_{j}(x)=A_{j}^{-1} \frac{\sin \left(2 \theta\left(x, k_{j}\right)\right)}{1+x},
$$

which we think of as vectors in the Hilbert space $\mathcal{H}=L^{2}([0, L],(1+x) d x)$. The normalization constants $A_{j}$ are chosen so that the vectors have norm one. Note that by Lemma 8.1,

$$
\left|\int_{0}^{L} \frac{\sin \left(2 \theta\left(x, k_{j}\right)\right)^{2}}{1+x} d x-\frac{1}{2} \log L\right| \leq C(I, B),
$$

and so $A_{j}^{2}=\frac{1+\sigma}{2} \log \epsilon^{-1}+O(1)$ (we denote by $O$ expressions which are bounded by constants depending only on $B, I, \sigma, \beta)$. Notice that (8.4) can be interpreted as

$$
\left|\left\langle V, e_{j}\right\rangle_{\mathcal{H}}\right|^{2} \geq \frac{a^{2}(1-\beta)^{2}}{10000(1+\sigma)} \log \epsilon^{-1}+O(1) .
$$

Moreover, by Lemma 8.1, (2.3) and the separation assumption, the vectors $e_{j}$ are almost orthogonal in the following sense:

$$
\left|\left\langle e_{l}, e_{j}\right\rangle_{\mathcal{H}}\right| \leq \frac{2}{1+\sigma} C(I, B) N^{-2}+O\left(\left(\log \epsilon^{-1}\right)^{-1}\right) .
$$

Now, by Lemma 4.4 of [12], if we have $N$ vectors $e_{j}$ satisfying $\alpha \equiv N \sup _{i \neq j}\left|\left\langle e_{i}, e_{j}\right\rangle_{\mathcal{H}}\right|$ $<1$, then for any vector $g$,

$$
\sum_{j=1}^{N}\left|\left\langle g, e_{j}\right\rangle_{\mathcal{H}}\right|^{2} \leq(1+\alpha)\|g\|_{\mathcal{H}}^{2} .
$$

Assume that $N$ is large enough so that $\frac{2}{1+\sigma} C(I, B) N^{-1}<1 / 2$. We are going to show that $N$ cannot exceed the right-hand side of (8.9) below, which we denote by $\tilde{N}(B, I, \sigma, \beta)$. Assume, on the contrary, that we can find $N=\tilde{N}(B, I, \sigma, \beta)+1$ resonant energies. Take $\epsilon$ sufficiently small so that the second term on the righthand side of (8.7) multiplied by $N$ is also smaller than $1 / 2$. 
Then Lemma 4.4 of [12] is applicable. Finally, note that $\|V\|_{\mathcal{H}}^{2} \leq(1+\sigma) B^{2} \log \epsilon^{-1}$. Combining (8.6), (8.7) and (8.8), we obtain

$$
N\left(\frac{a^{2}(1-\beta)^{2}}{10000(1+\sigma)} \log \epsilon^{-1}+O(1)\right) \leq 2(1+\sigma) B^{2} \log \epsilon^{-1} .
$$

But if $\epsilon$ is chosen sufficiently small (depending only on $I, B, \sigma$ and $\beta$ ), the above inequality implies

$$
N \leq\left(\frac{200(1+\sigma) B}{a(1-\beta)}\right)^{2} .
$$

Thus, if $\epsilon$ is sufficiently small, we get

$$
N \leq \max \left\{\frac{4}{1+\sigma} C(I, B),\left(\frac{200(1+\sigma) B}{a(1-\beta)}\right)^{2}\right\},
$$

which gives us a contradiction.

We are ready to give the proof of Theorem 1.3

Proof. Assume that the singular continuous spectrum is not empty. Fix an interval $I=[a, b], a>0$, such that the singular continuous part of the spectral measure gives a non-zero weight to it: $\mu_{s c}(I)=\delta_{0}>0$. Fix a small number $\epsilon_{0}>0$ to be determined later. At this point, we place two demands on $\epsilon_{0}$. First, $\epsilon_{0}<$ $\tilde{\epsilon}(B, I, \beta, \sigma)$ of Proposition 8.2. Second, fix some sufficiently large $N$, in particular $N>\tilde{N}(B, I, \beta, \sigma)$ appearing in Proposition 8.2. We ask that $\mu_{s c}(J)<\frac{1}{32} \delta_{0} N^{-3}$ for any interval $J \subset I$ such that $|J| \leq \epsilon_{0}^{N^{-2}}$. We say that an interval $J \subset I$ belongs to the scale $m$ if $|J|=\epsilon_{m} \equiv \epsilon_{0}^{m}$. We call an interval $J$ of scale $m$ singular if $\mu_{s c}(J) \geq \epsilon_{m}^{\beta}$. We call two intervals of the scale $m$ separated if the distance between their centers exceeds $2 \epsilon_{m}^{N^{-2}}$.

Lemma 8.3. There can be no more than $N$ separated singular intervals at each scale.

Proof. Assume that $J_{l}^{(m)}, l=1, \ldots, N$ are separated singular intervals of scale $m$. Take as usual $L_{m}=\epsilon_{m}^{-1-\sigma}$ and denote the spectral measure corresponding to the potential being cut off at $L_{m}$ by $\mu_{m}$. Denote by $2 J_{l}^{(m)}$ the interval with the same center as $J_{l}^{(m)}$ but twice its size. Then by (8.2) we have

$$
\mu_{m}\left(2 J_{l}^{(m)}\right) \geq \mu\left(J_{l}^{(m)}\right)-C \epsilon_{m}^{M} \geq \frac{1}{2} \epsilon_{m}^{\beta}
$$

provided $\epsilon_{0}$ is small enough. Combining (8.10) and (8.1), we see that there exist $k_{l}^{(m)} \in 2 J_{l}^{(m)}$ such that $R^{-2}\left(L_{m}, k_{l}^{(m)}\right) \geq \frac{1}{C(I)} \epsilon_{m}^{\beta-1}$. It is clear from (2.2) that this implies (8.4) at $k_{l}^{(m)}$ provided $\epsilon_{0}$ is small enough. Moreover, by the separation assumption, $\left|k_{l_{1}}^{(m)}-k_{l_{2}}^{(m)}\right|>\epsilon_{m}^{N^{-2}}$ for any $l_{1} \neq l_{2}$. By our choice of $\epsilon_{0}$, we can apply Proposition 8.2 and get a contradiction since $N>\tilde{N}$. Notice that we actually proved a stronger fact that the number of separated singular intervals cannot exceed $\tilde{N}$ of Proposition 8.2 .

Now we complete the proof of the main theorem. Define the set $S_{m}$ as a union of all singular intervals $J$ at scale $m$. It is easy to see that the set $S_{m}$ can be covered by at most $8 N$ intervals of size $\epsilon_{m}^{N^{-2}}$, or else we will be able to find more than $N$ separated singular intervals at scale $m$. The second condition we imposed on 
the size of $\epsilon_{0}$ ensures that for any $m, \mu_{s c}\left(S_{m}\right) \leq \frac{1}{4} N^{-2} \delta_{0}$. Consider $m \geq N^{2}$. The set $S_{m}$ can be covered by no more than $8 N$ intervals $J_{l}^{(m)}$ of size $\epsilon_{0}^{m N^{-2}}$. Denote by $\tilde{m}$ the integer part of $m N^{-2}, \tilde{m}=\left[m N^{-2}\right]$. Then any interval $J_{l}^{(m)}$ satisfying $\mu_{s c}\left(J_{l}^{(m)}\right)>\epsilon_{\tilde{m}}^{\beta}$ already belongs to $S_{\tilde{m}}$. Therefore, for any $m \geq N^{2}$, we have

$$
\mu_{s c}\left(S_{m} \backslash \bigcup_{l<m} S_{l}\right) \leq 8 N \epsilon_{\tilde{m}}^{\beta} .
$$

Combining the bounds we have yields

$$
\mu_{s c}\left(\bigcup_{m=1}^{\infty} S_{m}\right) \leq \frac{1}{4} \delta_{0}+\sum_{\tilde{m}=1}^{\infty} 8 N^{3} \epsilon_{0}^{\tilde{m} \beta} \leq \frac{1}{4} \delta_{0}+16 N^{3} \epsilon_{0} .
$$

Now we impose the final requirement on $\epsilon_{0}$, making sure that $\epsilon_{0}<\frac{1}{64 N^{3}} \delta_{0}$. Then (8.11) implies $\mu_{s c}\left(\bigcup_{m=1}^{\infty} S_{m}\right)<\frac{1}{2} \delta_{0}$. On the other hand, it is known that $\mu_{s c}$ can only be zero-dimensional for potentials satisfying our decay assumption [22, 4. Thus, $\mu_{s c}$ is supported on a set $S$ such that for any $k \in S$ and any $\alpha>0$,

$$
D^{\alpha} \mu_{s c}(k)=\limsup _{\delta \rightarrow 0} \frac{\mu(k-\delta, k+\delta)}{(2 \delta)^{\alpha}}=\infty .
$$

In particular, $S \subset \bigcup_{m=1}^{\infty} S_{m}$. But this gives a contradiction, via

$$
\delta_{0}=\mu_{s c}(S)=\mu_{s c}\left(\bigcup_{m=1}^{\infty} S_{m}\right) \leq \frac{1}{2} \delta_{0} .
$$

Acknowledgment. The author is grateful to Michael Christ and Barry Simon for useful discussions. The author also wishes to express his gratitude to François Germinet and Stephan de Bièvre for their hospitality at the Université de Lille I, and to Jan Janas and Sergei Naboko at the International Banach Center, where parts of this work have been carried out.

\section{REFERENCES}

[1] R. Carmona, Exponential localization in one-dimensional disordered systems, Duke Math. J. 49 (1982), 191-213. MR0650377 (84j:82082)

[2] R. Carmona and J. Lacroix, Spectral Theory of Random Schrödinger Operators, Boston: Birkhäuser, 1990. MR 1102675 (92k:47143)

[3] M. Christ and A. Kiselev, Absolutely continuous spectrum for one-dimensional Schrödinger operators with slowly decaying potentials: Some optimal results, J. Amer. Math. Soc. 11 (1998), 771-797. MR1621882 (99g:34166)

[4] — WKB and spectral analysis of one-dimensional Schrödinger operators with slowly varying potentials, Comm. Math. Phys. 218 (2001), 245-262. MR1828980 (2002e:34143)

[5] _ Scattering and wave operators for one-dimensional Schrödinger operators with slowly decaying nonsmooth potentials, Geom. Funct. Anal. 12 (2002), 1174-1234. MR1952927 (2003m:47019)

[6] E.A. Coddington and N. Levinson, Theory of Ordinary Differential Equations, McGraw-Hill, New York, 1955.

[7] H. Cycon, R. Froese, W. Kirsch and B. Simon, Schrödinger Operators, Springer-Verlag, 1987. MR0883643 (88g:35003)

[8] P. Deift and R. Killip, On the absolutely continuous spectrum of one-dimensional Schrödinger operators with square summable potentials, Commun. Math. Phys. 203 (1999), 341-347. MR.1697600 (2000c:34223) 
[9] S. Denisov, On the coexistence of absolutely continuous and singular continuous components of the spectral measure for some Sturm-Liouville operators with square summable potentials, J. Differential Equations 191 (2003), 90-104. MR.1973283(2004b:34219)

[10] F. Germinet, A. Kiselev and S. Tcheremchantsev, Transfer matrices and transport for 1D Schrödinger operators with singular spectrum, Annales de l'Institut Fourier, 54 (2004), 787830. MR2097423

[11] R. Killip and B. Simon, Sum rules for Jacobi matrices and their applications to spectral theory, Ann. of Math. 158 (2003), 253-321. MR.1999923 (2004f:47040)

[12] A. Kiselev, Y. Last, and B. Simon, Modified Prüfer and EFGP transforms and the spectral analysis of one-dimensional Schrödinger operators, Commun. Math. Phys. 194 (1998), 1-45. MR.1628290 (99g:34167)

[13] T. Kriecherbauer and C. Remling, Finite gap potentials and WKB asymptotics for onedimensional Schrödinger operators, Comm. Math. Phys. 223 (2001), 409-435. MR1864439 (2003a:81046)

[14] Y. Last, Quantum dynamics and decompositions of singular continuous spectra, J. Funct. Anal. 142 (1996), 406-445. MR1423040 (97k:81044)

[15] B. Levitan, Inverse Sturm-Liouville Problems, VNU Science Press, Utrecht 1987. MR0933088 (89b:34001)

[16] V. Marchenko, Sturm-Liouville Operators and Applications, Birkhäuser, Basel, 1986. MR 0897106 (88f:34034)

[17] S.N. Naboko, Dense point spectra of Schrödinger and Dirac operators, Theor.-math. 68 (1986), 18-28. MR0875178 (88h:81029)

[18] D. Pearson, Singular continuous measures in scattering theory, Comm. Math. Phys. 60 (1978), 13-36. MR0484145 (58:4076)

[19] M. Reed and B. Simon, Methods of Modern Mathematical Physics, I. Functional Analysis, Academic Press, New York, 1972. MR0493419 (58:12429a)

[20] - Methods of Modern Mathematical Physics, III. Scattering Theory, Academic Press, London-San Diego, 1979. MR0529429 (80m:81085)

[21] C. Remling, The absolutely continuous spectrum of one-dimensional Schrödinger operators with decaying potentials, Comm. Math. Phys. 193 (1998), 151-170. MR1620313 (99f:34123)

[22] _ Bounds on embedded singular spectrum for one-dimensional Schrödinger operators, Proc. Amer. Math. Soc. 128 (2000), no. 1, 161-171. MR.1637420(2000c:34225)

[23] _ Schrödinger operators with decaying potentials: some counterexamples, Duke Math. J. 105 (2000), no. 3, 463-496. MR.1801769 (2002e:81033)

[24] B. Simon, Schrödinger operators in the twenty-first century, Mathematical Physics 2000, 283-288, Imp. Coll. Press, London, 2000. MR1773049 (2001g:81071)

[25] _ Some Schrödinger operators with dense point spectrum, Proc. Amer. Math. Soc. 125 (1997), 203-208. MR1346989 (97c:34179)

[26] _ Operators with singular continuous spectrum. I. General operators, Ann. of Math. (2) 141 (1995), no. 1, 131-145. MR1314033 (96a:47038)

[27] J. von Neumann and E.P. Wigner, Über merkwürdige diskrete Eigenwerte, Z. Phys. 30 (1929), $465-467$

Department of Mathematics, University of Wisconsin, Madison, Wisconsin 53706-1388 E-mail address: kiselev@math.wisc.edu 